\title{
Discovery of novel 1,2,4-triazolo-1,2,4- triazines with thiomethylpyridine hinge binders as potent c-Met kinase inhibitors
}

Article

Accepted Version

Dadashpour, S., Kucukkilinc, T. T., Ayazgok, B., Hosseinimehr, S. J., Chippindale, A., Foroumadi, A. and Irannejad, H. (2019)

Discovery of novel 1,2,4-triazolo-1,2,4-triazines with thiomethylpyridine hinge binders as potent c-Met kinase inhibitors. Future Medicinal Chemistry, 11 (10). pp. 1119-1136. ISSN 1756-8927 doi: https://doi.org/10.4155/fmc-2018-0412 Available at https://centaur.reading.ac.uk/82834/

It is advisable to refer to the publisher's version if you intend to cite from the work. See Guidance on citing.

To link to this article DOI: http://dx.doi.org/10.4155/fmc-2018-0412

Publisher: Future Science Group

All outputs in CentAUR are protected by Intellectual Property Rights law, including copyright law. Copyright and IPR is retained by the creators or other copyright holders. Terms and conditions for use of this material are defined in the End User Agreement. 


\section{CentAUR}

Central Archive at the University of Reading

Reading's research outputs online 


\section{Discovery of novel 1,2,4-triazolo-1,2,4-triazines with thiomethylpyridine hinge binders as potent c-Met kinase inhibitors}

Sakineh Dadashpour ${ }^{1,2}$, Tuba Tuylu Kucukkilinc ${ }^{3}$, Beyza Ayazgok ${ }^{3}$, Seyed Jalal Hosseinimehr $^{4}$, Ann M Chippindale ${ }^{5}$, Alireza Foroumadi ${ }^{6} \&$ Hamid Irannejad*, $^{*}$

${ }^{1}$ Department of Medicinal Chemistry, Faculty of Pharmacy, Mazandaran University of Medical Sciences, Sari, Iran

${ }^{2}$ Student Research Committee, Mazandaran University of Medical Sciences, Sari, Iran

${ }^{3}$ Department of Biochemistry, Faculty of Pharmacy, Hacettepe University, Sihhiye, Ankara 06100, Turkey

${ }^{4}$ Department of Radiopharmacy, Faculty of Pharmacy, Mazandaran University of Medical Sciences, Sari, Iran

${ }^{5}$ Department of Chemistry, University of Reading, Whiteknights, Reading, Berks RG6 6AD, UK

${ }^{6}$ Department of Medicinal Chemistry, Faculty of Pharmacy \& Pharmaceutical Sciences Research Center, Tehran University of Medical Sciences, Tehran, Iran

*Author for correspondence: $\quad$ Tel.: $\quad 0098$ 9124572673, $\quad$ Fax: $0098 \quad 11$ 33543084; irannejadhamid@gmail.com

Running title: Triazolotriazines as c-Met kinase inhibitors 


\section{Graphical Abstract}

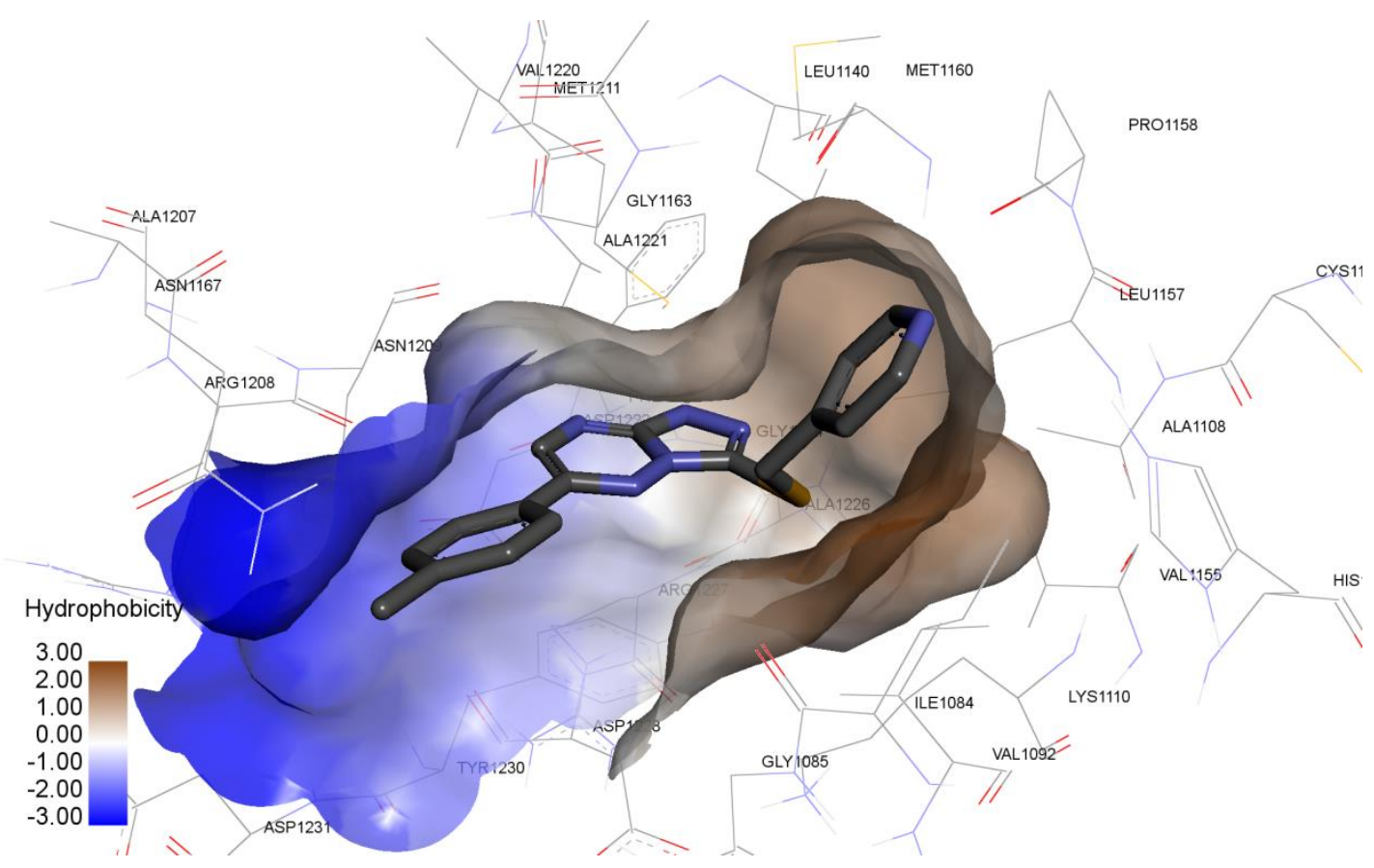

Compound $10 b$ was introduced as a potent c-Met kinase inhibitor with $\mathrm{IC}_{50}$ of $4.6 \mathrm{nM}$ and was more cytotoxic than crizotinib on HepG2 cells.

Aim: Mesenchymal-epithelial transition factor HGF/c-Met overactivation is involved in diverse human cancers. Herein, we report the synthesis and biological evaluation of thiomethylpyridine-linked triazolotriazines as c-Met kinase inhibitors.

Results: Compounds $\mathbf{1 0 b}$ and $11 \boldsymbol{e}$ were more potent than crizotinib on HepG2 cells with $\mathrm{IC}_{50}$ values of 0.74 and $0.71 \mu \mathrm{M}$ in the MTT assay, respectively. Interestingly, all of the target compounds displayed IC50 values in the range of 3.9-11.1 nM in the c-Met kinase inhibition assay which were lower than the value for crizotinib (11.1 nM).

Conclusion: Target compound $\mathbf{1 0 b}$ can be considered as a leading drug candidate due to its lower IC50 values than crizotinib in both HGF-induced proliferation and c-Met kinase inhibition assays. 
Keywords: Synthesis, [1,2,4]triazolo[4,3-b][1,2,4]triazine, Anticancer, c-Met kinase, Caspase 3/7, X-ray crystallography

\begin{abstract}
Mesenchymal-epithelial transition factor (c-Met) pertaining to receptor tyrosine kinases is a cell-surface receptor for hepatocyte growth factor (HGF). c-Met is widely distributed in the brain, human liver, gastrointestinal tract, and kidney. c-Met signaling leads to cell proliferation, migration, survival and embryonic morphogenesis [1]. HGF/c-Met over-activation is involved in diverse human tumors, such as glioblastomas, various carcinomas in breast, lung, liver and prostate, and in ovarian cancer [2]. Different strategies have been employed to regulate HGF/c-Met signaling pathway using HGF antagonists, c-Met monoclonal antibodies, anti-HGF antibodies and small-molecule c-Met inhibitors [3]. Small-molecule cMet inhibitors, on the basis of their binding modes, have been subdivided into three types, type I, II and III. Type I inhibitors occupy the ATP binding pocket in the form of a "U" conformation and display high selectivity on the c-Met enzyme. Type II inhibitors have extended conformation in the ATP binding pocket of c-Met and do not have selectivity over the c-MET enzyme [4, 5]. SGX-523 (Figure 1), a type I and selective c-MET inhibitor has showed excellent antitumor activity on the U87MG xenograft model [6]. Based on previous reports, triazolotriazines bearing 4-hydroxyphenylmethyl at the 3-position and Olinked triazolotriazines demonstrated superior c-met inhibitory activity (Figure 1, compounds 1 and 2) [7]. In the same compounds, the presence of a hydrogen-bond acceptor atom like nitrogen in the quinoline motif (Figure 1) was proved to constitute a crucial hydrogen bond with $\mathrm{NH}$ atom of Met1160 in the hinge binding region of the c-Met active site [8]. With respect to the importance of the electron-deficient triazolotriazine core in the formation of a strong $\pi$ - $\pi$ stacking interaction with Tyr-1230 in the c-Met activation loop, which confers potency and selectivity for c-Met inhibition against a wide range of
\end{abstract}


kinases, we designed and synthesized the 6-aryl-triazolotriazines, 10a-f and 11a-f (Figure 1), linked through thiomethylene to a 3- or 4-pyridyl ring. The flexible thiomethylene side chain containing the pyridine motif as a hinge binder ensures the formation of an essential hydrogen bond with Met1160. The effect of introducing the new thiomethylene linker to attach the hinge binder pyridine ring to the triazolotriazine core has been investigated for c-Met kinase inhibition and cell proliferation assays.

The chemical structure of synthesized titled compounds was confirmed by X-ray diffraction, ${ }^{1} \mathrm{H}$ and ${ }^{13} \mathrm{C}$ NMR, as well as Mass spectrometry. The anticancer activity of the target compounds was evaluated by HGF-induced proliferation assay against A549, HepG2, MCF7 and MDA-MB231 cell lines and compared to crizotinib as a selective c-MET inhibitor. The extent of cytotoxicity and apoptosis mediated by Caspase 3/7 activity were assessed and finally, the ability of the target compounds to inhibit c-Met kinase was evaluated and expressed as enzyme percent inhibition. 


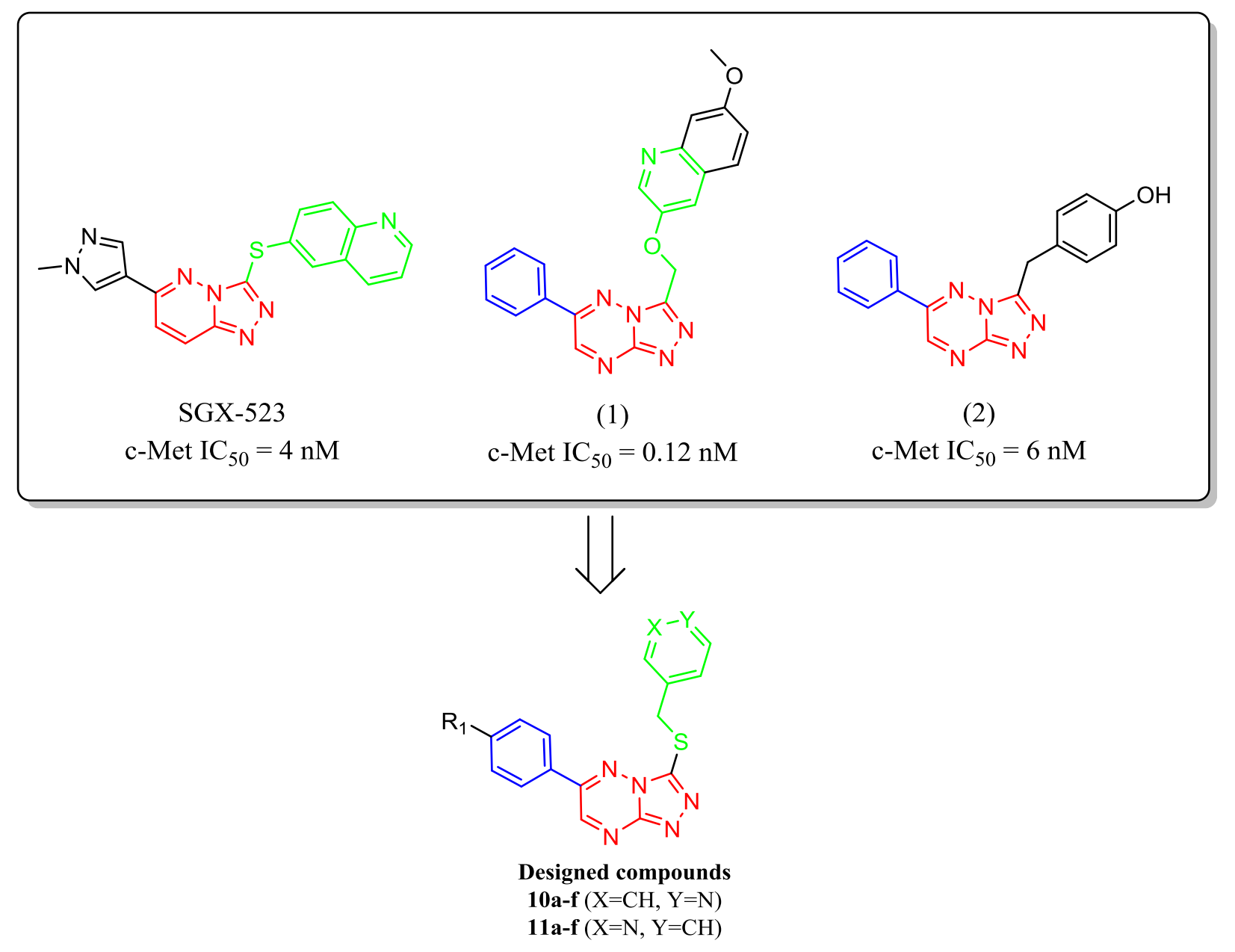

Figure 1. Schematic representation of designed triazolotriazines $\mathbf{1 0 a}-\boldsymbol{f}$ and $\mathbf{1 1 a} \boldsymbol{f}$, based on the previous cMet inhibitors.

\section{Experimental section}

\section{Material \& Methods}

All chemical reagents and solvents were purchased from Sigma-Aldrich or Merck suppliers and used without further purification. Crizotinib and HGF were purchased from Sigma. The progress of reactions was checked by Thin-layer chromatography (TLC) using TLC Silica gel $60 \mathrm{~F}_{254}$ (Merck, Darmstadt, Germany). A UV cabinet with wavelengths 254 and $360 \mathrm{~nm}$ was used for visualizing spots on the TLC plates. Column chromatography was performed using Silica gel 60 (0.040-0.063 mm), Merck, Germany. Melting points were measured in open capillaries on a Stuart Scientific apparatus and are uncorrected. IR spectra were recorded on a FT-IR Perkin 
Elmer spectrophotometer. NMR spectra were recorded on Bruker $500 \mathrm{MHz}$ spectrometer and tetramethylsilane (TMS) was used as the standard and chemical shifts are expressed in ppm units. Coupling constants are reported in Hertz $(\mathrm{Hz})$. Low resolution mass spectra were measured using a HP 5975 Mass Selective Detector (Agilent technologies). Elemental analyses for C, H and $\mathrm{N}$ were performed by using a $\mathrm{CHN}$ elemental analyzer (GmbH-Germany), and the results are within $\pm 0.4 \%$ of the theoretical values.

Preparation of methyl hydrazinecarbimidothioate hydroiodide.

A mixture of thiosemicarbazide $(1 \mathrm{mmol})$ and iodomethane $(10 \mathrm{mmol})$ in absolute ethanol were heated at $50^{\circ} \mathrm{C}$ for $3 \mathrm{~h}$. After cooling to r.t. a white precipitate was filtrated and air dried. Yield: 64\%.m.p. $138^{\circ} \mathrm{C}$.

\section{General procedure for the preparation of 2,2-dihydroxy-1-arylethanones 4 a- $f$}

To a suspension of $\mathrm{SeO}_{2}$ (1 equiv.) in a mixture of 1,4-dioxane and $\mathrm{H}_{2} \mathrm{O}$ (21:1) was added acetophenones 3a-f ( 1 equiv.) which was then heated at reflux for $2 \mathrm{~h}$. The reaction mixture was cooled to room temperature and filtered through Celite. The filtrate was concentrated in vacuo, water was added and then heated at reflux for $5 \mathrm{~h}$. Next, the reaction mixture was cooled in an ice bath to begin precipitation. The white precipitate obtained was filtered and dried in an oven.

2,2-Dihydroxy-1-phenylethanone (4a). Yield: 57\%. White powder. m.p. $83-85^{\circ} \mathrm{C}$. IR (KBr, $\mathrm{cm}^{-}$ 1): $3405(\mathrm{O}-\mathrm{H}), 1697(\mathrm{C}=\mathrm{O})$.

2,2-Dihydroxy-1-(p-tolyl)ethanone (4b). Yield: 55\%. White powder. m.p. $113-115^{\circ} \mathrm{C}$. IR $(\mathrm{KBr}$, $\mathrm{cm}^{-1}$ ): 3399 (O-H), 2915 (aliphatic C-H), $1687(\mathrm{C}=\mathrm{O}) .{ }^{1} \mathrm{H}$ NMR (500 MHz, CDCl 3 ) $\delta: 2.47$ (s, 3H), $7.34(\mathrm{~d}, J=8.2 \mathrm{~Hz}, 2 \mathrm{H}), 8.13(\mathrm{~d}, J=8.3 \mathrm{~Hz}, 2 \mathrm{H}), 9.68(\mathrm{~s}, 1 \mathrm{H})$.

2,2-Dihydroxy-1-(4-methoxyphenyl)ethanone (4c). Yield: 56\%. White powder. m.p. 111-114 ${ }^{\circ} \mathrm{C}$. IR (KBr, cm $\left.{ }^{-1}\right)$ : 3419 (O-H), 2987, 2944, 2846 (aliphatic C-H), 1682 (C=O).

2,2-Dihydroxy-1-(4-hydroxyphenyl)ethanone (4d). Yield: 50\%. White powder. m.p. 114-116 ${ }^{\circ} \mathrm{C}$. 
1-(4-Bromophenyl)-2,2-dihydroxyethanone (4e). Yield: $41 \%$. White powder. m.p. $131-133^{\circ} \mathrm{C}$. IR $\left(\mathrm{KBr}, \mathrm{cm}^{-1}\right)$ : $3378(\mathrm{O}-\mathrm{H}), 2974$ (aliphatic C-H), $1695(\mathrm{C}=\mathrm{O}) .{ }^{1} \mathrm{H}$ NMR (500 MHz, $\left.\mathrm{CDCl}_{3}\right) \delta$ : $3.99(\mathrm{~s}, 2 \mathrm{H}), 5.93(\mathrm{~s}, 1 \mathrm{H}), 7.70(\mathrm{~d}, J=8.3 \mathrm{~Hz}, 2 \mathrm{H}), 8.11(\mathrm{~d}, J=8.4 \mathrm{~Hz}, 2 \mathrm{H}), 9.65(\mathrm{~s}, 1 \mathrm{H})$.

1-(4-Chlorophenyl)-2,2-dihydroxyethanone (4f). Yield: $35 \%$. White powder. m.p. $96-98^{\circ} \mathrm{C}$. IR $\left(\mathrm{KBr}, \mathrm{cm}^{-1}\right): 3374(\mathrm{O}-\mathrm{H}), 2978$ (aliphatic C-H), $1697(\mathrm{C}=\mathrm{O}) .{ }^{1} \mathrm{H}$ NMR $\left(500 \mathrm{MHz}, \mathrm{CDCl}_{3}\right) \delta$ : $7.51(\mathrm{~d}, J=8.3 \mathrm{~Hz}, 2 \mathrm{H}), 8.18(\mathrm{~d}, J=7.7 \mathrm{~Hz}, 2 \mathrm{H}), 9.64(\mathrm{~s}, 1 \mathrm{H})$.

General procedure for the preparation of 2,2-diethoxy-1-arylethanones 5 a-f

To a solution of 2,2-dihydroxy-1-arylethanones, $4 a-f$ ( 1 equiv.) in dichloromethane, $p$ toluenesulfonic acid (PTSA, 0.05 equiv.) and triethyl orthoformate (2 equiv.) were added and heated at reflux for $2 \mathrm{~h}$. The solvent was removed under reduced pressure and the residue was chromatographed on silica gel (230-400 mesh) using chloroform as eluent.

2,2-Diethoxy-1-phenylethanone (5a). Yield: 90\%. Yellow oil. IR $\left(\mathrm{KBr}, \mathrm{cm}^{-1}\right)$ : 3068 (aromatic CH), 2979, 2932, 2882 (aliphatic C-H), $1689(\mathrm{C}=\mathrm{O}) .{ }^{1} \mathrm{H}$ NMR (500 MHz, $\left.\mathrm{CDCl}_{3}\right) \delta: 1.26(\mathrm{t}, J=$ $7.0 \mathrm{~Hz}, 6 \mathrm{H}), 3.64-3.70(\mathrm{~m}, 2 \mathrm{H}), 3.75-3.81(\mathrm{~m}, 2 \mathrm{H}), 5.30(\mathrm{~s}, 1 \mathrm{H}), 7.45-7.49(\mathrm{~m}, 2 \mathrm{H})$, 7.75-7.60 $(\mathrm{m}, 1 \mathrm{H}), 8.17-8.19(\mathrm{~m}, 2 \mathrm{H})$.

2,2-Diethoxy-1-(p-tolyl)ethanone (5b). Yield: $80 \%$. Yellow oil. ${ }^{1} \mathrm{H}$ NMR (500 $\left.\mathrm{MHz}, \mathrm{CDCl}_{3}\right) \delta$ : $1.26(\mathrm{t}, J=7.0 \mathrm{~Hz}, 6 \mathrm{H}), 2.42(\mathrm{~s}, 3 \mathrm{H}), 3.63-3.70(\mathrm{~m}, 2 \mathrm{H}), 3.73-3.80(\mathrm{~m}, 2 \mathrm{H}), 5.29(\mathrm{~s}, 1 \mathrm{H}), 7.27(\mathrm{~d}$, $J=8.1 \mathrm{~Hz}, 2 \mathrm{H}), 8.08(\mathrm{~d}, J=8.2 \mathrm{~Hz}, 2 \mathrm{H})$.

2,2-Diethoxy-1-(4-methoxyphenyl)ethanone (5c). Yield: $91 \%$. Yellow oil. ${ }^{1} \mathrm{H}$ NMR $(500 \mathrm{MHz}$, $\left.\mathrm{CDCl}_{3}\right) \delta: 1.25(\mathrm{t}, J=7.0,6 \mathrm{H}), 3.62-3.68(\mathrm{~m}, 2 \mathrm{H}), 3.73-3.80(\mathrm{~m}, 2 \mathrm{H}), 3.88(\mathrm{~s}, 3 \mathrm{H}), 5.25(\mathrm{~s}, 1 \mathrm{H})$, $6.94(\mathrm{~d}, J=9 \mathrm{~Hz}, 2 \mathrm{H}), 8.17(\mathrm{~d}, J=8.9 \mathrm{~Hz}, 2 \mathrm{H})$.

2,2-Diethoxy-1-(4-hydroxyphenyl)ethanone (5d). Yield: 90\%. Pale yellow oil. The title compound $\boldsymbol{5} \boldsymbol{d}$ was purified by column chromatography, eluting with $5 \%$ ethylacetate in chloroform. ${ }^{1} \mathrm{H}$ NMR $\left(500 \mathrm{MHz}, \mathrm{CDCl}_{3}\right) \delta: 1.25(\mathrm{t}, J=7.0 \mathrm{~Hz}, 6 \mathrm{H}), 3.64-3.70(\mathrm{~m}, 2 \mathrm{H}), 3.72-$ $3.78(\mathrm{~m}, 2 \mathrm{H}), 5.33(\mathrm{~s}, 1 \mathrm{H}), 6.93(\mathrm{~d}, J=8.8 \mathrm{~Hz}, 2 \mathrm{H}), 8.12(\mathrm{~d}, J=8.8 \mathrm{~Hz}, 2 \mathrm{H})$.

1-(4-Bromophenyl)-2,2-diethoxyethanone (5e). Yield: 88\%. Pale yellow oil. IR $\left(\mathrm{KBr}, \mathrm{cm}^{-1}\right)$ : 2978, 2931, 2882 (aliphatic C-H), 1693 (C=O). ${ }^{1} \mathrm{H}$ NMR (500 MHz, $\left.\mathrm{CDCl}_{3}\right) \delta: 1.10$ (t, $J=6.9$ 
$\mathrm{Hz}, 6 \mathrm{H}), 3.47-3.52(\mathrm{~m}, 2 \mathrm{H}), 3.61-3.65(\mathrm{~m}, 2 \mathrm{H}), 5.04(\mathrm{~s}, 1 \mathrm{H}), 7.43(\mathrm{~d}, J=7.9 \mathrm{~Hz}, 2 \mathrm{H}), 7.92(\mathrm{~d}, J$ $=7.2 \mathrm{~Hz}, 2 \mathrm{H})$.

1-(4-Chlorophenyl)-2,2-diethoxyethanone (5f). Yield: $85 \%$. Pale yellow oil. . IR $\left(\mathrm{KBr}, \mathrm{cm}^{-1}\right)$ : 3441 (aromatic C-H), 2979, 2931, 2896 (aliphatic C-H), 1693 (C=O).

General procedure for the preparation of 3-(methylthio)-6-aryl-1,2,4-triazines $\mathbf{6 a - f}$.

To a solution of 2,2-diethoxy-1-arylethanones, $5 \boldsymbol{a}-\boldsymbol{c}$ and $\mathbf{5 e - f}$ (1 equiv.) in absolute ethanol were added PTSA (0.05 equiv.) and thiosemicarbazide (1 equiv.) and stirred at r.t. for $8 \mathrm{~h}$. The completion of the reaction was monitored by TLC. After completion, 1.1 equiv. of methyl iodide was added to the mixture and stirring was continued overnight. The solvent was removed under reduced pressure and acetic acid was added to the obtained residue and heated at $60{ }^{\circ} \mathrm{C}$ overnight with stirring. Finally, the resulting solution was concentrated in vacuo and the crude product was purified by column chromatography (silica gel, $230-400$ mesh) eluting with chloroform.

3-(Methylthio)-6-phenyl-1,2,4-triazine (6a). Yield: 50\%. Brown powder. m.p. 93-95 ${ }^{\circ} \mathrm{C} .{ }^{1} \mathrm{H}$ NMR $\left(500 \mathrm{MHz}, \mathrm{CDCl}_{3}\right) \delta: 2.82(\mathrm{~s}, 3 \mathrm{H}), 7.61-7.65(\mathrm{~m}, 3 \mathrm{H}), 8.12-8.14(\mathrm{~m}, 2 \mathrm{H}), 8.87(\mathrm{~s}, 1 \mathrm{H}) ; \mathrm{MS}$ : $(\mathrm{m} / \mathrm{z}, \%): 203.1\left(\mathrm{M}^{+}, 30\right), 175.1(10), 102.2(100), 76.1(25)$.

3-(Methylthio)-6-(p-tolyl)-1,2,4-triazine (6b). Yield: 30\%. Brown powder. m.p. $119-120^{\circ} \mathrm{C} .{ }^{1} \mathrm{H}$ NMR (500 MHz, $\left.\mathrm{CDCl}_{3}\right) \delta: 2.46(\mathrm{~s}, 3 \mathrm{H}), 2.74(\mathrm{~s}, 3 \mathrm{H}), 7.37$ (d, $\left.J=8 \mathrm{~Hz}, 2 \mathrm{H}\right), 7.96(\mathrm{~d}, J=8.2$ Hz, 2H), 8.77 (s, 1H); MS: (m/z, \%): $217.2\left(\mathrm{M}^{+}, 31\right), 116.2$ (100), 89.1 (14).

6-(4-Methoxyphenyl)-3-(methylthio)-1,2,4-triazine (6c). Yield: 40\%. Brown powder. m.p. 140$143^{\circ} \mathrm{C} .{ }^{1} \mathrm{H}$ NMR $\left(500 \mathrm{MHz}, \mathrm{CDCl}_{3}\right) \delta: 2.74(\mathrm{~s}, 3 \mathrm{H}), 3.90$ (s, 3H), 7.07 (d, J=8.9 Hz, 2H), 8.02 (d, $J=8.8 \mathrm{~Hz}, 2 \mathrm{H}), 8.75$ (s, 1H); MS: (m/z, \%): $233.1\left(\mathrm{M}^{+}, 82\right), 132.2$ (100), 117.1 (74), 89.1 (66).

4-(3-(Methylthio)-1,2,4-triazin-6-yl)phenol (6d). Yield: 41\%. To a suspension of 2,2-diethoxy-1(4-hydroxyphenyl)ethanone, $5 \boldsymbol{d}$ (1 equiv.) in absolute ethanol, PTSA (0.05 equiv.) and methyl hydrazinecarbimidothioate hydroiodide (1 equiv.) were added and stirred at r.t. for $8 \mathrm{~h}$. The solution was concentrated under vacuum, then acetic acid was added and heated at $60{ }^{\circ} \mathrm{C}$ overnight with stirring. The crude product was purified on a silica gel column, eluting with $5 \%$ ethylacetate in chloroform to give the yellow powder. m.p. $147-150{ }^{\circ} \mathrm{C} .{ }^{1} \mathrm{H}$ NMR $(500 \mathrm{MHz}$, 
DMSO-d $)_{6} \delta: 2.65(\mathrm{~s}, 3 \mathrm{H}), 6.94(\mathrm{~d}, J=8.7 \mathrm{~Hz}, 2 \mathrm{H}), 8.01(\mathrm{~d}, J=8.7 \mathrm{~Hz}, 2 \mathrm{H}), 9.13(\mathrm{~s}, 1 \mathrm{H}), 10.07$ (s, 1H); MS: (m/z, \%): $219.1\left(\mathrm{M}^{+}, 86\right), 118.2$ (100), 89.1 (65).

6-(4-Bromophenyl)-3-(methylthio)-1,2,4-triazine (6e). Yield: 54\%. The residue was crystallized from ethanol to aff ord $\boldsymbol{\sigma} \boldsymbol{e}$ as brown crystals. m.p. $157-159{ }^{\circ} \mathrm{C} .{ }^{1} \mathrm{H}$ NMR $\left(500 \mathrm{MHz}, \mathrm{CDCl}_{3}\right) \delta$ : $2.74(\mathrm{~s}, 3 \mathrm{H}), 7.70(\mathrm{~d}, J=8.5 \mathrm{~Hz}, 2 \mathrm{H}), 7.94(\mathrm{~d}, J=8.5 \mathrm{~Hz}, 2 \mathrm{H}), 8.77$ (s, 1H); MS: (m/z, \%): 281.0 $\left(\mathrm{M}^{+}, 19\right), 283.1(\mathrm{M}+2,19), 180.1$ (100), $101.1(57)$.

6-(4-Chlorophenyl)-3-(methylthio)-1,2,4-triazine (6f). Yield: 37\%. Brown powder. m.p. 155$157^{\circ} \mathrm{C} .{ }^{1} \mathrm{H} \mathrm{NMR}\left(500 \mathrm{MHz}, \mathrm{CDCl}_{3}\right) \delta: 2.74(\mathrm{~s}, 3 \mathrm{H}), 7.53(\mathrm{~d}, J=8.6 \mathrm{~Hz}, 2 \mathrm{H}), 8.01(\mathrm{~d}, J=8.6 \mathrm{~Hz}$, 2H), 8.77 (s, 1H); MS: (m/z, \%): $237.1\left(\mathrm{M}^{+}, 20\right), 239.1$ (M+2, 7.7), 136.2 (100), 101.1 (29).

General procedure for the preparation of 3-(methylsulfonyl)-6-aryl-1,2,4-triazines 7a-f

To a suspension of 3-(methylthio)-6-aryl-1,2,4-triazines, 6 a-f (1 equiv.) in THF was added potassium peroxymonosulfate (oxone ${ }^{\circledR}, 1$ equiv.) in water, and mixture was stirred at r.t. for $2 \mathrm{~h}$ (water/THF, 1:1, v/v). After completion of the reaction, it was partially concentrated in vacuo to $50 \%$ volume. The precipitate was filtered and washed with water and dried in oven. The crude product was crystallized from ethanol to afford light yellow crystals.

3-(Methylsulfonyl)-6-phenyl-1,2,4-triazine (7a). Yield: 66\%. m.p. 180-183 ${ }^{\circ}$. IR ( $\left.\mathrm{KBr}, \mathrm{cm}^{-1}\right)$ : 3461, 3058 (aromatic C-H), 2916 (aliphatic C-H), 1598, 1537 (S=O). ${ }^{1} \mathrm{H}$ NMR (500 MHz, $\left.\mathrm{CDCl}_{3}\right) \delta: 3.15$ (s, 3H), 7.63-7.64 (m, 3H), 8.16-8.18 (m, 2H), 9.20 (s, 1H); MS: (m/z, \%): 235.1 $\left(\mathrm{M}^{+}, 2\right), 219.1$ (14), 128.1 (73), 102.1 (100), 77.1 (40).

3-(Methylsulfonyl)-6-(p-tolyl)-1,2,4-triazine (7b). Yield: 43\%. m.p. 200-202 ${ }^{\circ} \mathrm{C} . \mathrm{IR}\left(\mathrm{KBr}, \mathrm{cm}^{-1}\right)$ : 3399, 3065, 3002 (aromatic C-H), 2919 (aliphatic C-H), 1607, 1554 (S=O). ${ }^{1} \mathrm{H}$ NMR (500 MHz, $\left.\mathrm{CDCl}_{3}\right) \delta: 2.48(\mathrm{~s}, 3 \mathrm{H}), 3.13(\mathrm{~s}, 3 \mathrm{H}), 7.42(\mathrm{~d}, J=8.0 \mathrm{~Hz}, 2 \mathrm{H}), 8.07(\mathrm{~d}, J=8.2 \mathrm{~Hz}, 2 \mathrm{H}), 9.16(\mathrm{~s}$, 1H); MS: (m/z, \%): $249.1\left(\mathrm{M}^{+}, 2\right), 237.1$ (10), 190.1 (11), 136.0 (100), 116.1 (100).

6-(4-Methoxyphenyl)-3-(methylsulfonyl)-1,2,4-triazine (7c). Yield: 83\%. m.p. $144-145^{\circ} \mathrm{C}$. IR $\left(\mathrm{KBr}, \mathrm{cm}^{-1}\right.$ ): 3451, 3000 (aromatic C-H), 2967, 2931, 2838 (aliphatic C-H), 1605, 1578 (S=O). ${ }^{1} \mathrm{H}$ NMR (500 MHz, $\left.\mathrm{CDCl}_{3}\right) \delta: 3.13(\mathrm{~s}, 3 \mathrm{H}), 3.94(\mathrm{~s}, 3 \mathrm{H}), 7.13(\mathrm{~d}, J=8.9 \mathrm{~Hz}, 2 \mathrm{H}), 8.16(\mathrm{~d}, J=$ $8.9 \mathrm{~Hz}, 2 \mathrm{H}), 9.14$ (s, 1H); MS: (m/z, \%): $265.1\left(\mathrm{M}^{+}, 2\right), 249.1$ (12), 174.1 (20), 158.1 (59), 132.1 (100). 
4-(3-(Methylsulfonyl)-1,2,4-triazin-6-yl)phenol (7d). Yield: 60\%. m.p. 195-197 ${ }^{\circ} \mathrm{C} .{ }^{1} \mathrm{H}$ NMR $(500$ MHz, DMSO-d6) $\delta: 3.01$ (s, 3H), 7.00 (d, $J=8.7 \mathrm{~Hz}, 2 \mathrm{H}), 8.16(\mathrm{~d}, J=8.7 \mathrm{~Hz}, 2 \mathrm{H}), 9.52(\mathrm{~s}, 1 \mathrm{H})$, 10.30 (s, 1H); MS: (m/z, \%): 251.9 (M+2, 2), 179.9 (26), 132.1 (33), 93.0 (49), 64.0 (100).

6-(4-Bromophenyl)-3-(methylsulfonyl)-1,2,4-triazine (7e). Yield: 85\%. m.p. 189-191 ${ }^{\circ} \mathrm{C} . \quad \mathrm{IR}$ $\left(\mathrm{KBr}, \mathrm{cm}^{-1}\right): 3459,3085,3047$ (aromatic C-H), $1586(\mathrm{~S}=\mathrm{O}) .{ }^{1} \mathrm{H}$ NMR $\left(500 \mathrm{MHz}, \mathrm{CDCl}_{3}\right) \delta: 3.15$ (s, 3H), 7.78 (d, $J=8.6 \mathrm{~Hz}, 2 \mathrm{H}), 8.06$ (d, $J=8.6 \mathrm{~Hz}, 2 \mathrm{H}), 9.18$ (s, 1H); MS: (m/z, \%): 313.0 $\left(\mathrm{M}^{+}, 2\right), 315.0(\mathrm{M}+2,2), 297.0$ (9), 206.0 (41), 180.1 (100), 127.1 (98), 101.1 (57).

6-(4-Chlorophenyl)-3-(methylsulfonyl)-1,2,4-triazine (7f). Yield: 88\%. m.p. $210-212{ }^{\circ} \mathrm{C} .{ }^{1} \mathrm{H}$ NMR (500 MHz, $\left.\mathrm{CDCl}_{3}\right) \delta: 3.15$ (s,3H), $7.61(\mathrm{~d}, J=8.6 \mathrm{~Hz}, 2 \mathrm{H}), 8.14(\mathrm{~d}, J=8.7 \mathrm{~Hz}, 2 \mathrm{H}), 9.18$ (s, 1H); MS: (m/z, \%): 269.1 (M+1, 0.5), 253.1 (8), 162.1 (46), 136.1 (100), 101.1 (19).

General procedure for the preparation of 3-hydrazinyl-6-aryl-1,2,4-triazines 8a-f

To a suspension of 3-(methylsulfonyl)-6-aryl-1,2,4-triazines $7 a-f(1 \mathrm{mmol})$ in THF (11 ml) was added $0.53 \mathrm{ml}$ of hydrazine monohydrate and the resulting mixture stirred at r.t. for $2 \mathrm{~h}$. The precipitated product was filtered and air dried.

3-Hydrazinyl-6-phenyl-1,2,4-triazine (8a). Yield: 86\%. Yellow powder. m.p. 192-194 ${ }^{\circ}$. IR $\left(\mathrm{KBr}, \mathrm{cm}^{-1}\right.$ ): 3275, $3218(\mathrm{~N}-\mathrm{H}), 3055$ (aromatic C-H), 2965 (aliphatic C-H). ${ }^{1} \mathrm{H}$ NMR (500 MHz, DMSO-d 6 ) $\delta: 4.46$ (brs, 2H), $7.46(\mathrm{t}, J=7.3 \mathrm{~Hz}, 1 \mathrm{H}), 7.52(\mathrm{t}, J=7.5 \mathrm{~Hz}, 2 \mathrm{H}), 8.02(\mathrm{~d}, J=7.2$ Hz, 2H), 8.87 (s, 1H); MS: (m/z, \%): $187.2\left(\mathrm{M}^{+}, 80\right), 116(13), 102.2$ (100).

3-Hydrazinyl-6-(p-tolyl)-1,2,4-triazine (8b). Yield: 70\%. Yellow powder. m.p. $173-174^{\circ} \mathrm{C}$. I IR $\left(\mathrm{KBr}, \mathrm{cm}^{-1}\right):$ 3432, $3233(\mathrm{~N}-\mathrm{H}), 3043$ (aromatic C-H). ${ }^{1} \mathrm{H}$ NMR (500 MHz, DMSO-d 6 ) $\delta: 2.36(\mathrm{~s}$, 3H), 4.47 (brs, 2H), 7.32 (d, $J=8.1 \mathrm{~Hz}, 2 \mathrm{H}), 7.91$ (d, $J=8.2 \mathrm{~Hz}, 2 \mathrm{H}), 8.82$ (brs, 1H), 8.85 (s, 1H); MS: (m/z, \%): $201.2\left(\mathrm{M}^{+}, 84\right), 130.1$ (8), 116.2 (100), 89.1 (12).

3-Hydrazinyl-6-(4-methoxyphenyl)-1,2,4-triazine (8c). Yield: 65\%. Yellow powder. m.p. 213$215^{\circ} \mathrm{C}$. IR (KBr, cm $\left.{ }^{-1}\right): 3461,3254(\mathrm{~N}-\mathrm{H}), 3039$ (aromatic C-H), 2963, 2840 (aliphatic C-H). ${ }^{1} \mathrm{H}$

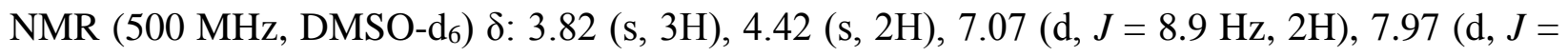
$8.8 \mathrm{~Hz}, 2 \mathrm{H}), 8.74$ (brs, 1H), 8.83 (s, 1H); MS: (m/z, \%): $217.2\left(\mathrm{M}^{+}, 72\right), 132.2$ (100), 117.0 (38), $89.1(32)$. 
4-(3-Hydrazinyl-1,2,4-triazin-6-yl)phenol (8d). Yield: 64\%. The yellow needle crystals were obtained by recrystallization from ethanol. m.p. $243-245^{\circ} \mathrm{C} .{ }^{1} \mathrm{H}$ NMR $\left(500 \mathrm{MHz}, \mathrm{DMSO}-\mathrm{d}_{6}\right) \delta$ : 4.38 (brs, 2H), 6.88 (d, $J=8.6 \mathrm{~Hz}, 2 \mathrm{H}), 7.84$ (d, $J=8.6 \mathrm{~Hz}, 2 \mathrm{H}), 8.67$ (brs, 1H), 8.77 (s, 1H).

6-(4-Bromophenyl)-3-hydrazinyl-1,2,4-triazine (8e). Yield: 90\%. Yellow powder. m.p. 228$230^{\circ} \mathrm{C} . \mathrm{IR}\left(\mathrm{KBr}, \mathrm{cm}^{-1}\right): 3438,3249(\mathrm{~N}-\mathrm{H}) .{ }^{1} \mathrm{H}$ NMR $(500 \mathrm{MHz}$, DMSO-d 6 ) $\delta: 4.48$ (s, 2H), 7.71 (d, $J=8.5 \mathrm{~Hz}, 2 \mathrm{H}), 7.98$ (d, $J=8.6 \mathrm{~Hz}, 2 \mathrm{H}), 8.89$ (s, 1H), 8.96 (brs, 1H); MS: (m/z, \%): 265.0 (M+, 35), 267.0 (M+2, 35), 250.0 (5), 182.0 (100), 101.0 (52).

6-(4-Chlorophenyl)-3-hydrazinyl-1,2,4-triazine (8f). Yield: 80\%. Yellow powder. m.p. 230$232^{\circ} \mathrm{C}$. IR (KBr, cm $\left.{ }^{-1}\right): 3301,3224(\mathrm{~N}-\mathrm{H}), 3042$ (aromatic C-H), 2969 (aliphatic C-H). ${ }^{1} \mathrm{H}$ NMR $\left(500 \mathrm{MHz}, \mathrm{DMSO}_{\mathrm{d}}\right.$ ) $\delta: 4.48$ (brs, 2H), 7.57 (d, $\left.J=8.6 \mathrm{~Hz}, 2 \mathrm{H}\right), 8.05(\mathrm{~d}, J=8.6 \mathrm{~Hz}, 2 \mathrm{H}), 8.89$ (s, 1H), 8.95 (brs, 1H).

General procedure for the preparation of 6-aryl-[1,2,4]triazolo[4,3-b][1,2,4]triazine-3-thiols $9 a-f$

To a suspension of 3-hydrazinyl-6-aryl-1,2,4-triazines 8 a-f $(1 \mathrm{mmol})$ in ethanol $(15 \mathrm{ml})$, were added successively potassium hydroxide $(2 \mathrm{~N}, 1.3 \mathrm{ml})$ and carbon disulfide $(1.3 \mathrm{ml})$ and the mixture was refluxed for $1 \mathrm{~h}$. After completion, the reaction mixture was cooled to r.t. and concentrated in vacuo. $1 \mathrm{~N}$ aqueous potassium hydroxide was added to the residue, heated and sonicated. Then solution was filtered and the filtrate was acidified to $\mathrm{pH} 2-3$ with $1 \mathrm{~N}$ aqueous $\mathrm{HCl}$ to precipitate the product. The resulting precipitate was filtered, washed with water and dried in oven. It was used in the next step without further purification.

6-Phenyl-[1,2,4]triazolo[4,3-b][1,2,4]triazine-3-thiol $(\mathbf{9 a})$. Yield: 88\%. Light orange powder.

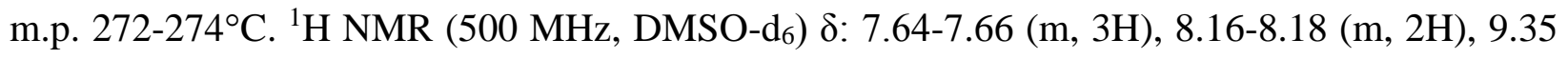
(s, 1H), 14.94 (s, 1H); MS: (m/z, \%): 229.1 (M+1 100), 197.1 (5), 116.1 (22), 104.1 (51), 89.1 (15).

6-(p-Tolyl)-[1,2,4]triazolo[4,3-b][1,2,4]triazine-3-thiol (9b). Yield: 60\%. Light orange powder. m.p. $254-257^{\circ} \mathrm{C} .{ }^{1} \mathrm{H}$ NMR (500 MHz, DMSO-d 6 ) $\delta: 2.43$ (s, 3H), 7.45 (d, $\left.J=8 \mathrm{~Hz}, 2 \mathrm{H}\right), 8.09$ (d, $J=8.2 \mathrm{~Hz}, 2 \mathrm{H}), 9.34$ (s, 1H), 14.88 (s, 1H); MS: (m/z, \%): $243.1\left(\mathrm{M}^{+}, 100\right), 229.1$ (3), 155.1 (10), $130.1(26), 118.1(60), 91.1(26)$. 
6-(4-Methoxyphenyl)-[1,2,4]triazolo[4,3-b][1,2,4]triazine-3-thiol (9c). Yield: 82\%. Light orange powder. m.p. $232-234^{\circ} \mathrm{C} .{ }^{1} \mathrm{H}$ NMR $(500 \mathrm{MHz}$, DMSO-d 6 ) $\delta: 3.88$ (s, 3H), 7.19 (d, $J=8.9 \mathrm{~Hz}$, 2H), $8.16(\mathrm{~d}, J=8.9 \mathrm{~Hz}, 2 \mathrm{H}), 9.34(\mathrm{~s}, 1 \mathrm{H}), 14.84(\mathrm{~s}, 1 \mathrm{H})$.

4-(3-Mercapto-[1,2,4]triazolo[4,3-b][1,2,4]triazin-6-yl)phenol (9d). Yield: 71\%. Brick red powder. m.p. $260-262^{\circ} \mathrm{C} .{ }^{1} \mathrm{H}$ NMR $\left(500 \mathrm{MHz}, \mathrm{DMSO}-\mathrm{d}_{6}\right) \delta: 6.99(\mathrm{~d}, J=8.7 \mathrm{~Hz}, 2 \mathrm{H}), 8.06(\mathrm{~d}, J$ $=8.8 \mathrm{~Hz}, 2 \mathrm{H}), 9.30(\mathrm{~s}, 1 \mathrm{H}), 14.81(\mathrm{~s}, 1 \mathrm{H})$.

6-(4-Bromophenyl)-[1,2,4]triazolo[4,3-b][1,2,4]triazine-3-thiol (9e). Yield: 73\%. Light orange powder. m.p. $257-259^{\circ} \mathrm{C} .{ }^{1} \mathrm{H}$ NMR $\left(500 \mathrm{MHz}, \mathrm{DMSO}-\mathrm{d}_{6}\right) \delta: 7.87(\mathrm{~d}, J=8.6 \mathrm{~Hz}, 2 \mathrm{H}), 8.12(\mathrm{~d}, J$ $=8.6 \mathrm{~Hz}, 2 \mathrm{H}), 9.35(\mathrm{~s}, 1 \mathrm{H}), 14.95(\mathrm{~s}, 1 \mathrm{H}) ; \mathrm{MS}:(\mathrm{m} / \mathrm{z}, \%): 307.0\left(\mathrm{M}^{+}, 8\right), 309(\mathrm{M}+2,8), 267.0$ (100), 196 (69), 149.9 (36), 102.0 (31), 88.0 (24).

6-(4-Chlorophenyl)-[1,2,4]triazolo[4,3-b][1,2,4]triazine-3-thiol (9f). Yield: 90\%. Light orange powder. m.p. $247-249^{\circ} \mathrm{C} .{ }^{1} \mathrm{H}$ NMR $\left(500 \mathrm{MHz}, \mathrm{DMSO}_{-} \mathrm{d}_{6}\right) \delta: 7.73(\mathrm{~d}, J=8.7 \mathrm{~Hz}, 2 \mathrm{H}), 8.20$ (d, $J$ $=8.7 \mathrm{~Hz}, 2 \mathrm{H}), 9.35$ (s, 1H), $14.95(\mathrm{~s}, 1 \mathrm{H}) ; \mathrm{MS}:(\mathrm{m} / \mathrm{z}, \%): 263.1\left(\mathrm{M}^{+}, 8\right), 219.1(33), 168.0(88)$, 149.0 (100), $136.0(30), 89.1(38)$.

\section{General procedure for the preparation of final derivatives}

To a suspension of 6-aryl-[1,2,4]triazolo[4,3-b][1,2,4]triazine-3-thiols 9a- $\boldsymbol{f}$ (1 equiv.) and 1.1 equiv. of 3- or 4-(chloromethyl)pyridine hydrochloride in DMSO, was added 2.5 equiv. of potassium carbonate which was then stirred at r.t. for $3 \mathrm{~h}$. Chloroform was added to the resulting mixture and washed with water $(\times 3)$, then dried over $\mathrm{Na}_{2} \mathrm{SO}_{4}$ and concentrated to give the crude product as a brown oil. Final products were purified by column chromatography (silica gel, 230400 mesh) eluting with chloroform/methanol (1\%).

6-Phenyl-3-((pyridin-4-ylmethyl)thio)-[1,2,4]triazolo[4,3-b][1,2,4]triazine (10a)

Yield: 30\%; m.p. $130-132^{\circ} \mathrm{C} .{ }^{1} \mathrm{H}$ NMR $\left(500 \mathrm{MHz}, \mathrm{CDCl}_{3}\right) \delta: 4.59$ (s, $\left.2 \mathrm{H}\right), 7.38(\mathrm{~d}, J=5.9 \mathrm{~Hz}$, 2H), 7-56-7.61 (m, 3H), $7.98(\mathrm{dt}, J=1.5,6.6 \mathrm{~Hz}, 2 \mathrm{H}), 8.50(\mathrm{~d}, J=5.8 \mathrm{~Hz}, 2 \mathrm{H}), 8.95(\mathrm{~s}, 1 \mathrm{H}) ;{ }^{13} \mathrm{C}$ NMR (125 MHz, $\left.\mathrm{CDCl}_{3}\right)$ \&: 34.6, 124.1, 127.6, 129.8, 131.1, 132.3, 144.8, 145.7, 146.0, 148.2, 148.8, 150.2; MS: (m/z, \%): $320.1\left(\mathrm{M}^{+}, 92\right), 287.1$ (9), 229.1 (100), 190.1 (31), 104.1 (64), 77.1 (35). Anal. Calcd for $\mathrm{C}_{16} \mathrm{H}_{12} \mathrm{~N}_{6} \mathrm{~S}$ : C, 59.98; H, 3.78; N, 26.23. Found: C, 59.89; H, 3.83; N, 26.29. 
3-((Pyridin-4-ylmethyl)thio)-6-(p-tolyl)-[1,2,4]triazolo[4,3-b][1,2,4]triazine (10b)

Yield: 35\%; m.p. $187-188^{\circ} \mathrm{C} .{ }^{1} \mathrm{H}$ NMR $\left(500 \mathrm{MHz}, \mathrm{CDCl}_{3}\right) \delta$ : 2.44 (s, 3H), 4.58 (s, 2H), 7.36$7.38(\mathrm{~m}, 4 \mathrm{H}), 7.88(\mathrm{~d}, J=8.2 \mathrm{~Hz}, 2 \mathrm{H}), 8.50(\mathrm{dd}, J=1.5,6 \mathrm{~Hz}, 2 \mathrm{H}), 8.93(\mathrm{~s}, 1 \mathrm{H}) ;{ }^{13} \mathrm{C}$ NMR $(125$ $\left.\mathrm{MHz}, \mathrm{CDCl}_{3}\right) \delta: 21.7,34.6,124.1,127.4,128.2,130.5,143.1,144.7,145.7,146.1,148.2,148.8$, 150.2; MS: (m/z, \%): 334.1 ( $\left.\mathrm{M}^{+}, 100\right), 301.1$ (10), 256.1 (8), 224.1 (8), 190.0 (47), 116.1 (47). Anal. Calcd for $\mathrm{C}_{17} \mathrm{H}_{14} \mathrm{~N}_{6} \mathrm{~S}: \mathrm{C}, 61.06 ; \mathrm{H}, 4.22 ; \mathrm{N}, 25.13$. Found: C, 61.19; H, 4.24; N, 25.09.

6-(4-Methoxyphenyl)-3-((pyridin-4-ylmethyl)thio)-[1,2,4]triazolo[4,3-b][1,2,4]triazine (10c)

Yield: 37\%; m.p. $148-150{ }^{\circ} \mathrm{C} .{ }^{1} \mathrm{H}$ NMR $\left(500 \mathrm{MHz}, \mathrm{CDCl}_{3}\right) \delta: 3.88(\mathrm{~s}, 3 \mathrm{H}), 4.56(\mathrm{~s}, 2 \mathrm{H}), 7.05(\mathrm{~d}$, $J=8.9 \mathrm{~Hz}, 2 \mathrm{H}), 7.37(\mathrm{~d}, J=5.9 \mathrm{~Hz}, 2 \mathrm{H}), 7.94(\mathrm{~d}, J=8.8 \mathrm{~Hz}, 2 \mathrm{H}), 8.49(\mathrm{~d}, J=6 \mathrm{~Hz}, 2 \mathrm{H}), 8.91$ $(\mathrm{s}, 1 \mathrm{H}) ;{ }^{13} \mathrm{C} \mathrm{NMR}\left(125 \mathrm{MHz}, \mathrm{CDCl}_{3}\right) \delta: 34.6,55.79,115.3,123.3,124.1,129.2,144.6,145.8$, 146.0, 147.7, 148.8, 150.2, 163.0; MS: (m/z, \%): $350.1\left(\mathrm{M}^{+}, 100\right), 317.1$ (8), 215.1 (23), 190.0 (46), 146.1 (46), 132.1 (56). Anal. Calcd for $\mathrm{C}_{17} \mathrm{H}_{14} \mathrm{~N}_{6} \mathrm{OS}$ : C, 58.27; H, 4.03; N, 23.98. Found: C, $58.35 ; \mathrm{H}, 4.05 ; \mathrm{N}, 24.05$.

4-(3-((Pyridin-4-ylmethyl)thio)-[1,2,4]triazolo[4,3-b][1,2,4]triazin-6-yl)phenol (10d)

Yield: $15 \%$; m.p. $211-213{ }^{\circ} \mathrm{C} .{ }^{1} \mathrm{H}$ NMR (500 MHz, DMSO-d 6$)$ ) 4.45 (s, 2H), 6.89 (d, $J=8.5$ Hz, 2H), 7.29 (d, $J=4.7 \mathrm{~Hz}, 2 \mathrm{H}), 7.94$ (d, $J=8.6 \mathrm{~Hz}, 2 \mathrm{H}), 8.37$ (brs, 2H), 9.22 (s, 1H), 10.28 (s, $1 \mathrm{H}) ;{ }^{13} \mathrm{C}$ NMR $(125 \mathrm{MHz}$, DMSO-d 6$) \delta: 34.5,116.8,122.5,124.5,130.1,143.2,147.1,148.3$, 148.6, 149.3, 150.2, 161.6; MS: (m/z, \%): $336.1\left(\mathrm{M}^{+}, 15\right), 187.1$ (31), 119.0 (42), 93.1 (100). Anal. Calcd for $\mathrm{C}_{16} \mathrm{H}_{12} \mathrm{~N}_{6} \mathrm{OS}$ : C, 57.13; H, 3.60; N, 24.98. Found: C, 57.02; H, 3.66; N, 24.90. 6-(4-Bromophenyl)-3-((pyridin-4-ylmethyl)thio)-[1,2,4]triazolo[4,3-b][1,2,4]triazine (10e)

Yield: $30 \%$; m.p. $158-160^{\circ} \mathrm{C} .{ }^{1} \mathrm{H}$ NMR $\left(500 \mathrm{MHz}, \mathrm{CDCl}_{3}\right) \delta: 4.53$ (s, $\left.2 \mathrm{H}\right), 7.32(\mathrm{~d}, J=5.6 \mathrm{~Hz}$, $2 \mathrm{H}), 7.66(\mathrm{~d}, J=8.3 \mathrm{~Hz}, 2 \mathrm{H}), 7.81(\mathrm{~d}, J=8.3 \mathrm{~Hz}, 2 \mathrm{H}), 8.44(\mathrm{~d}, J=5.4 \mathrm{~Hz}, 2 \mathrm{H}), 8.85(\mathrm{~s}, 1 \mathrm{H}) ;{ }^{13} \mathrm{C}$ NMR (125 MHz, $\left.\mathrm{CDCl}_{3}\right) \delta: 34.5,124.1,127.4,128.9,129.9,133.1,145.0,145.4,145.6,147.3$, 148.6, 150.2; MS: (m/z, \%): $398\left(\mathrm{M}^{+}, 58\right), 400$ (M+2, 60), 367.0 (9), 334.1 (22), 215.1 (18), 190.0 (100), 92.1 (73). Anal. Calcd for $\mathrm{C}_{16} \mathrm{H}_{11} \mathrm{BrN}_{6} \mathrm{~S}: \mathrm{C}, 48.13$; H, 2.78; N, 21.05. Found: $\mathrm{C}$, 48.22; H, 2.71; N, 21.12. 
6-(4-Chlorophenyl)-3-((pyridin-4-ylmethyl)thio)-[1,2,4]triazolo[4,3-b][1,2,4]triazine (10f)

Yield: 20\%; m.p. $151-153{ }^{\circ} \mathrm{C} .{ }^{1} \mathrm{H}$ NMR $\left(500 \mathrm{MHz}, \mathrm{CDCl}_{3}\right) \delta: 4.54(\mathrm{~s}, 2 \mathrm{H}), 7.33(\mathrm{~d}, J=4.9 \mathrm{~Hz}$, 2H), $7.51(\mathrm{~d}, J=8.4 \mathrm{~Hz}, 2 \mathrm{H}), 7.88(\mathrm{~d}, J=8.4 \mathrm{~Hz}, 2 \mathrm{H}), 8.45(\mathrm{~d}, J=4.9 \mathrm{~Hz}, 2 \mathrm{H}), 8.68(\mathrm{~s}, 1 \mathrm{H})$; MS: (m/z, \%): $354.1\left(\mathrm{M}^{+}, 100\right), 356.1$ (M+2, 39), 321.1 (22), 263.0 (58), 190.0 (85), 163.0 (27), 136.0 (85), 92.1 (77). Anal. Calcd for $\mathrm{C}_{16} \mathrm{H}_{11} \mathrm{ClN}_{6} \mathrm{~S}$ : C, 54.16; H, 3.13; N, 23.69. Found: C, 54.25; H, 3.18; N, 23.61.

6-Phenyl-3-((pyridin-3-ylmethyl)thio)-[1,2,4]triazolo[4,3-b][1,2,4]triazine (11a)

Yield: 38\%; m.p. $145-147{ }^{\circ} \mathrm{C} .{ }^{1} \mathrm{H}$ NMR $\left(500 \mathrm{MHz}, \mathrm{CDCl}_{3}\right) \delta$ : 4.62 (s, 2H), 7.20 (dd, $J=4.8,7.8$ $\mathrm{Hz}, 1 \mathrm{H}), 7.56-7.61(\mathrm{~m}, 3 \mathrm{H}), 7.82(\mathrm{dt}, J=1.9,7.8 \mathrm{~Hz}, 1 \mathrm{H}), 7.98(\mathrm{dt}, J=1.7,6.5 \mathrm{~Hz}, 2 \mathrm{H}), 8.46$ $(\mathrm{dd}, J=1.5,4.7 \mathrm{~Hz}, 1 \mathrm{H}), 8.66(\mathrm{~d}, J=2.0 \mathrm{~Hz}, 1 \mathrm{H}), 8.95(\mathrm{~s}, 1 \mathrm{H}) ;{ }^{13} \mathrm{C} \mathrm{NMR}\left(125 \mathrm{MHz}, \mathrm{CDCl}_{3}\right) \delta$ : 33.2, 123.6, 127.6, 129.8, 131.1, 132.3, 132.6, 136.9, 145.0, 146.0, 148.2, 148.7, 149.3,150.3; MS: (m/z, \%): $320.2\left(\mathrm{M}^{+}, 18\right), 287.1$ (20), 242.1 (8), 190.1 (56), 116.1 (29), 92.1 (100). Anal. Calcd for $\mathrm{C}_{16} \mathrm{H}_{12} \mathrm{~N}_{6} \mathrm{~S}$ : C, 59.98; H, 3.78; N, 26.23. Found: C, 60.09; H, 3.84; N, 26.16.

3-((Pyridin-3-ylmethyl)thio)-6-(p-tolyl)-[1,2,4]triazolo[4,3-b][1,2,4]triazine (11b)

Yield: 30\%; m.p. 173-175 ${ }^{\circ} \mathrm{C} .{ }^{1} \mathrm{H}$ NMR $\left(500 \mathrm{MHz}, \mathrm{CDCl}_{3}\right) \delta: 2.43$ (s, 3H), 4.60 (s, 2H), 7.18 (dd, $J=4.8,7.8 \mathrm{~Hz}, 1 \mathrm{H}), 7.34(\mathrm{~d}, J=8.0 \mathrm{~Hz}, 2 \mathrm{H}), 7.80(\mathrm{dt}, J=1.8,7.8 \mathrm{~Hz}, 1 \mathrm{H}), 7.86(\mathrm{~d}, J=8.2 \mathrm{~Hz}$, $2 \mathrm{H}), 8.44(\mathrm{~d}, J=4.5 \mathrm{~Hz}, 1 \mathrm{H}), 8.64(\mathrm{~s}, 1 \mathrm{H}), 8.92(\mathrm{~s}, 1 \mathrm{H}) ;{ }^{13} \mathrm{C} \mathrm{NMR}\left(125 \mathrm{MHz}, \mathrm{CDCl}_{3}\right) \delta: 21.7$, 33.2, 123.6, 127.4, 128.2, 130.5, 132.6, 136.9, 143.1, 144.9, 146.1, 148.1, 148.8, 149.2, 150.3; MS: (m/z, \%): $334.1\left(\mathrm{M}^{+}, 36\right), 301.1$ (29), 256.1 (12), 215.1 (15), 190.0 (73), 130.1 (35), 116.1 (62), 92.1 (100). Anal. Calcd for $\mathrm{C}_{17} \mathrm{H}_{14} \mathrm{~N}_{6} \mathrm{~S}$ : C, 61.06; H, 4.22; N, 25.13. Found: C, 61.14; H, $4.16 ; \mathrm{N}, 25.06$.

6-(4-Methoxyphenyl)-3-((pyridin-3-ylmethyl)thio)-[1,2,4]triazolo[4,3-b][1,2,4]triazine (11c)

Yield: 34\%; m.p. $146-148^{\circ} \mathrm{C} .{ }^{1} \mathrm{H}$ NMR (500 MHz, $\left.\mathrm{CDCl}_{3}\right) \delta: 3.87$ (s, 3H), 4.59 (s, 2H), 7.03 (d, $J=8.8 \mathrm{~Hz}, 2 \mathrm{H}), 7.18(\mathrm{dd}, J=4.8,7.7 \mathrm{~Hz}, 1 \mathrm{H}), 7.80(\mathrm{~d}, J=7.8 \mathrm{~Hz}, 1 \mathrm{H}), 7.93(\mathrm{dd}, J=1.8,8.8$ $\mathrm{Hz}, 2 \mathrm{H}), 8.43(\mathrm{~d}, J=4.7 \mathrm{~Hz}, 1 \mathrm{H}), 8.63(\mathrm{~s}, 1 \mathrm{H}), 8.91(\mathrm{~s}, 1 \mathrm{H}) ;{ }^{13} \mathrm{C} \mathrm{NMR}\left(125 \mathrm{MHz}, \mathrm{CDCl}_{3}\right) \delta$ : 33.2, 55.7, 115.2, 123.3, 123.6, 129.2, 132.6, 136.9, 144.7, 146.0, 147.7, 148.7, 149.2, 150.3, 163.0; MS: (m/z, \%): $350.1\left(\mathrm{M}^{+}, 42\right), 317.1$ (23), 272.1 (12), 190.0 (62), 132.1 (75), 92.1 (100). Anal. Calcd for $\mathrm{C}_{17} \mathrm{H}_{14} \mathrm{~N}_{6} \mathrm{OS}$ : C, 58.27; H, 4.03; N, 23.98. Found: C, 58.32; H, 4.08; N, 23.90. 
4-(3-((Pyridin-3-ylmethyl)thio)-[1,2,4]triazolo[4,3-b][1,2,4]triazin-6-yl)phenol (11d)

Yield: 25\%; m.p. 268-270 ${ }^{\circ} \mathrm{C} .{ }^{1} \mathrm{H}$ NMR (500 MHz, DMSO-d $)$ ) $8: 4.61$ (s, 2H), 7.02 (d, $J=8.7$ $\mathrm{Hz}, 2 \mathrm{H}), 7.33(\mathrm{dd}, J=4.7,7.7 \mathrm{~Hz}, 1 \mathrm{H}), 7.83(\mathrm{dd}, J=1.5,7.8 \mathrm{~Hz}, 1 \mathrm{H}), 8.07$ (d, $J=8.7 \mathrm{~Hz}, 2 \mathrm{H})$, $8.44(\mathrm{~d}, J=4 \mathrm{~Hz}, 1 \mathrm{H}), 8.59(\mathrm{~s}, 1 \mathrm{H}), 9.33(\mathrm{~s}, 1 \mathrm{H}) ;{ }^{13} \mathrm{C} \mathrm{NMR}\left(125 \mathrm{MHz}, \mathrm{CDCl}_{3}\right) \delta: 32.5,116.2$, 121.8, 123.3, 129.4, 133.3, 136.4, 142.8, 147.7, 147.8, 148.4, 148.6, 149.8, 161.0; MS: (m/z, \%): $336.1\left(\mathrm{M}^{+}, 2\right), 292.1$ (9),174.1 (27), 119.0 (88), 92.1 (100). Anal. Calcd for $\mathrm{C}_{16} \mathrm{H}_{12} \mathrm{~N}_{6} \mathrm{OS}: \mathrm{C}$, 57.13; H, 3.60; N, 24.98. Found: C, 57.18; H, 3.56; N, 24.92.

6-(4-Bromophenyl)-3-((pyridin-3-ylmethyl)thio)-[1,2,4]triazolo[4,3-b][1,2,4]triazine (11e)

Yield: 37\%; m.p. $176-178^{\circ} \mathrm{C} .{ }^{1} \mathrm{H}$ NMR $\left(500 \mathrm{MHz}, \mathrm{CDCl}_{3}\right) \delta$ : $4.63(\mathrm{~s}, 2 \mathrm{H}), 7.21$ (dd, $J=4.8,7.8$ $\mathrm{Hz}, 1 \mathrm{H}), 7.72(\mathrm{~d}, J=8.6 \mathrm{~Hz}, 2 \mathrm{H}), 7.82(\mathrm{dt}, J=1.9,7.8 \mathrm{~Hz}, 1 \mathrm{H}), 7.87(\mathrm{~d}, J=8.6 \mathrm{~Hz}, 2 \mathrm{H}), 8.46$ (dd, $J=1.5,4.8 \mathrm{~Hz}, 1 \mathrm{H}), 8.67(\mathrm{~d}, J=2.1 \mathrm{~Hz}, 1 \mathrm{H}), 8.91(\mathrm{~s}, 1 \mathrm{H}) ;{ }^{13} \mathrm{C} \mathrm{NMR}\left(125 \mathrm{MHz}, \mathrm{CDCl}_{3}\right) \delta$ : 33.2, 123.6, 127.4, 128.9, 130.0, 132.5, 133.1, 136.9, 145.2, 145.4, 147.3, 148.6, 149.3, 150.3; MS: (m/z, \%): $398\left(\mathrm{M}^{+}, 8\right), 400(\mathrm{M}+2,8), 365.1$ (8), 281.0 (4), 215.1 (14), 190.0 (63), 92.1 (100). Anal. Calcd for $\mathrm{C}_{16} \mathrm{H}_{11} \mathrm{BrN}_{6} \mathrm{~S}: \mathrm{C}, 48.13 ; \mathrm{H}, 2.78 ; \mathrm{N}, 21.05$. Found: C, 48.10; H, 2.84; N, 21.10 .

6-(4-Chlorophenyl)-3-((pyridin-3-ylmethyl)thio)-[1,2,4]triazolo[4,3-b][1,2,4]triazine (11f)

Yield: $40 \%$; m.p. $158-160^{\circ} \mathrm{C} .{ }^{1} \mathrm{H}$ NMR $\left(500 \mathrm{MHz}, \mathrm{CDCl}_{3}\right) \delta: 4.62(\mathrm{~s}, 2 \mathrm{H}), 7.20(\mathrm{dd}, J=4.9,7.7$ $\mathrm{Hz}, 1 \mathrm{H}), 7.55(\mathrm{~d}, J=8.6 \mathrm{~Hz}, 2 \mathrm{H}), 7.81(\mathrm{dt}, J=1.8,7.9 \mathrm{~Hz}, 1 \mathrm{H}), 7.94(\mathrm{~d}, J=8.6 \mathrm{~Hz}, 2 \mathrm{H}), 8.46$ $(\mathrm{d}, J=4.6 \mathrm{~Hz}, 1 \mathrm{H}), 8.66(\mathrm{~s}, 1 \mathrm{H}), 8.91(\mathrm{~s}, 1 \mathrm{H}) ;{ }^{13} \mathrm{C} \mathrm{NMR}\left(125 \mathrm{MHz}, \mathrm{CDCl}_{3}\right)$ 8: 33.2, 123.6, 128.8, 129.5, 130.1, 132.5, 136.9, 138.9, 145.2, 145.4, 147.2, 148.6, 149.3, 150.3; MS: (m/z, \%):

$354\left(\mathrm{M}^{+}, 12\right), 356$ (M+2, 4), 321.1 (12), 240.0 (13), 215.1 (22), 190.1 (46), 92.1 (100). Anal.

Calcd for $\mathrm{C}_{16} \mathrm{H}_{11} \mathrm{ClN}_{6} \mathrm{~S}: \mathrm{C}, 54.16 ; \mathrm{H}, 3.13 ; \mathrm{N}, 23.69$. Found: C, 54.19; H, 3.17; N, 23.77.

\section{X-ray crystallography}

A crystal of compound 11c was prepared by slow evaporation in acetonitrile and mounted under Paratone-N oil and flash cooled to $150 \mathrm{~K}$ in a stream of nitrogen in an Oxford Cryostream cooler. Single-crystal X-ray intensity data (Table 1) were collected using an Agilent Gemini S Ultra diffractometer $(\mathrm{Cu} \mathrm{K \alpha}$ radiation $(\lambda=1.54180 \AA))$. The data were reduced within the CrysAlisPro software. [9] The structure was solved using the program Superflip [10] and all non-hydrogen atoms located. Least-squares refinements on $F$ were carried out using the CRYSTALS suite of programs. [11] The non-hydrogen atoms were refined anisotropically. All hydrogen atoms were 
located using difference Fourier maps. Each hydrogen atom connected to carbon was placed geometrically with a C-H distance of $0.95 \AA$ and a $U$ iso of 1.2 times the value of Uequiv of the parent $\mathrm{C}$ atom. The positions of these hydrogen atoms were then refined with riding constraints. [12]

\section{HGF-induced proliferation assay}

The anti-proliferative activities of compounds and positive control crizotinib (Sigma, PZ0191) were evaluated against MCF-7, MDA-MB-231, HepG2 and A549 cell lines using MTT assay. The cancer cell lines were cultured in Dulbecco's modified eagle's medium (DMEM) supplemented with $10 \% \mathrm{FBS}, \% 1 \mathrm{~L}$-glutamine and $\% 0.5$ antibiotics at $37{ }^{\circ} \mathrm{C}$ in a humidified atmosphere containing $5 \% \mathrm{CO}_{2}$. Cancer cells were seeded in 96-well plates at 5000 cells/well. After 24 hours' incubation, cells were starved in FBS-free medium containing $0.1 \%$ BSA and recombinant human HGF (40 ng/ml) (Sigma, H5791) for $24 \mathrm{~h}$, then treated with test compounds at different concentrations for 72 hours at $37{ }^{\circ} \mathrm{C}$. After incubation, $10 \mu \mathrm{L}$ MTT reagent (5 $\mathrm{mg} / \mathrm{ml}$ ) was added to each well. MTT formazan crystals were dissolved by the addition of 100 $\mu \mathrm{L}$ DMSO. Relative cell viability was estimated by comparing the absorbance values at $690 \mathrm{~nm}$ (for absorbance of MTT formazan) and 570nm (for the reference wavelength) of the treated group and untreated control group using a Biotek Power Wave XS microplate reader. $\mathrm{IC}_{50}$ values were calculated using GraphPad Prism software. [13, 14]

\section{Caspase 3/7 Activity Assay}

MCF-7, MDA-MB-231, HepG2 and A549 cells were seeded at a density of 1 x $10^{3}$ cells per well in Lumox (Sarstedt) 384-well plates. After cells were challenged with recombinant human HGF (40 ng/ml) (Sigma, H5791) for $24 \mathrm{~h}$, the test compounds were added at $20 \mu \mathrm{M}$ concentration and the cells were incubated for an additional 24 hours at $37^{\circ} \mathrm{C}$. The ApoTox-Glo Triplex Assay (Promega, USA) was performed to estimate viability, cytotoxicity and caspase-3/7 activity following the manufacturer's instructions. Briefly $5 \mu \mathrm{L}$ viability/cytotoxicity reagent were added to the wells and after 1 hour of incubation at $37^{\circ} \mathrm{C}$, viability $\left(400_{\mathrm{ex}} / 505_{\mathrm{em}}\right)$ and cytotoxicity $\left(485_{\mathrm{ex}} / 520_{\mathrm{em}}\right)$ were measured. Later $25 \mu \mathrm{L}$ Caspase-Glo reagent was added to the wells and the plate is incubated for an hour at room temperature. Luminescence was measured using a multimode plate reader (BMG Labtech Omega FLUOstar). Caspase 3/7 activity results were normalized well-to-well by the ratio of the number of live cells to the number of dead cells. [15]

\section{c-Met inhibition assay}

Met Kinase Enzyme System (V3361, Promega, USA) with ADP-Glo assay was used for the cMet inhibition assay. It was performed in duplicate to screen kinase inhibitory activities of compounds at $20 \mu \mathrm{M}, 5 \mu \mathrm{M}, 0,5 \mu \mathrm{M}, 0.05 \mu \mathrm{M}$ and $0.5 \mathrm{nM}$ concentrations according to the manufacturer's instructions. Briefly reactions were set up in 384-well Lumox (Sarstedt) plates containing kinase buffer, $10 \mu \mathrm{M}$ ATP, $0.2 \mu \mathrm{g} / \mu \mathrm{l}$ poly (Glu, Tyr) 4:1 peptide substrate and $8 \mathrm{ng}$ cMet kinase for 1 hour at room tempature. ADP formed from kinase reactions were measured by ADP-Glo kinase assay. $5 \mu \mathrm{L}$ of ADP-Glo ${ }^{\mathrm{TM}}$ reagent was added to the wells to stop the kinase reaction and plate is incubated for $40 \mathrm{~min}$ at room temperature. After adding $10 \mu \mathrm{L}$ of kinase 
detection reagent and 30 min of additional incubation; luminescence was measured using a multimode plate reader (BMG Labtech Omega FLUOstar). The percentage of inhibition (I\%) was estimated by comparing the kinase activities of samples to vehicle. $\mathrm{IC}_{50}$ values of the compounds were determined from percent kinase inhibition values between $20 \mu \mathrm{M}-0.5 \mathrm{nM}$ compound concentrations using Graphpad Prism 5 software.

\section{Molecular docking study}

A docking study was performed employing the AutoDock 4.2 software with the AutoDock Tools (ADT) interface. Compounds $\mathbf{1 0 c}$ and $11 \boldsymbol{c}$ were docked into the binding site of c-met tyrosine kinase (PDB code: $3 Z X Z$ ). At first, for the preparation of protein, all the water and ligands were removed and the protein structure was optimized and the Kollman charge was computed. The compound's structure was then optimized and Gasteiger-Huckel charges were added. The Lamarckian genetic algorithm was utilized for the docking search. A grid box was generated by Autogrid 4.2. Autogrid and autodock files were created by the following parameters: Grid center: $X=19.775, Y=83.506, Z=4.432$, Grid spacing $=0.375$, number of points: $60 \times 60 \times 60$, number of evaluations: $2,500,000$, population size: 150 , GA run: 30 .

\section{Results \& Discussion}

\section{Chemistry}

The synthetic procedure for the title compounds is depicted in Figure 3. Oxidation of acetophenones (3a-f) by using selenium dioxide in a mixture of 1,4-dioxane and water in refluxing mode afforded corresponding 2,2-dihydroxy-1-arylethanones (4a-f) [16]. Subsequently compounds (4a-f) were reacted with triethyl orthoformate in the presence of $p$-toluenesulfonic acid (PTSA) in dichloromethane under reflux to give corresponding acetal intermediates (5a-f) [7]. The triazine ring closure was carried out in three successive steps for compounds $6 \boldsymbol{a}-\boldsymbol{c}$ and $\boldsymbol{6} \boldsymbol{e}-\boldsymbol{f}$. At the first step, reaction of acetal intermediates (5a-f) with thiosemicarbazide in the presence of PTSA in ethanol at room temperature led to the imine formation, then, methylation of the thiosemicarbazide thiol group was performed using iodomethane, and finally 1,2,4-triazine ring closure was done in acetic acid [17]. In order to prepare compound 6d, the acetal intermediate $\mathbf{5 d}$ was reacted with methyl hydrazinecarbimidothioate hydroiodide in the presence of PTSA in ethanol and then stirred in acetic acid at $60^{\circ} \mathrm{C}$ [18]. Next, the 3-methylthio group was oxidized by oxone in tetrahydrofuran and water at room temperature to afford 7a-f [19]. The 3hydrazinyl intermediates (8a-f) were prepared by replacement of the 3-methylsulfonyl group with a hydrazine group [17] and eventually it was cyclized in the presence of carbon disulfide in 
ethanol to give 3-mercapto-[1,2,4]triazolo[4,3-b][1,2,4]triazines 9a-f [20]. Final products, 10a-f and 11a-f, were prepared by adding commercially available 3- or 4-(chloromethyl)pyridine hydrochloride to 3-mercapto-1,2,4-triazolo-1,2,4-triazines 9a-f in dry DMSO and potassium carbonate [21]. Exploring ${ }^{1} \mathrm{H}$ NMR spectra of compounds 10a-f and 11a-f (NMR spectra in Supplementary material) shows that aliphatic $\mathrm{SCH}_{2}$ protons appeared as singlet and are located at 4.45-4.59 and 4.59-4.63 ppm respectively. Notably, a singlet appeared at 8.85-8.95 ppm for compounds 10a-f and 11a-f is attributed to the $\mathrm{C} 7-\mathrm{H}$ proton of the triazine ring (Figure 2), except in 10d and 11d, where the same proton has been deshielded to 9.22 and $9.33 \mathrm{ppm}$, respectively. The ${ }^{1} \mathrm{H}-\mathrm{NMR}$ spectra of compounds 10a-f show 4 doublets in the aromatic part of each spectrum, of which two of them belong to the para-substituted aryl ring located at position 6 of triazolotriazine nucleus. Basically, the other two doublets in the form of an AB quartet at 7.3 and 8.4-8.5 ppm correspond to the pyridine ring for compounds 10a-f. For example, the spectrum of 6-(4-bromophenyl)-3-((pyridin-4-ylmethyl)thio)-[1,2,4]triazolo[4,3b] [1,2,4]triazine (10e) revealed two singlet signals in 8.85 and $4.53 \mathrm{ppm}$ attributed to $\mathrm{C}-\mathrm{H}$ in the triazolotriazine ring and $\mathrm{S}-\mathrm{CH}_{2}$, respectively. The $\mathrm{AB}$ quartet signals at 7.32 and $8.44 \mathrm{ppm}$ correspond to pyridine ring protons and the $\mathrm{AB}$ quartet signals at 7.66 and $7.81 \mathrm{ppm}$ are related to 4-bromophenyl ring protons. ${ }^{13} \mathrm{C}$-NMR spectrum of 10e displayed one signal at $34.5 \mathrm{ppm}$ referring to $\mathrm{S}-\mathrm{CH}_{2}$, and 11 signals ranging from 124.1-150.2 ppm attributed to carbons in aromatic rings.

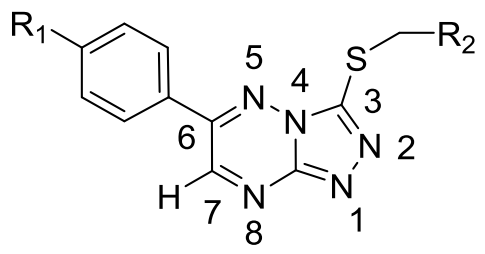

Figure 2. Atom numbering of $[1,2,4]$ triazolo[4,3-b][1,2,4]triazine nucleus in IUPAC nomenclature.

In the ${ }^{1} \mathrm{H}-\mathrm{NMR}$ spectra of compounds 11a-f, the aryl ring substituted at position 6 of the triazolotriazine nucleus revealed two doublets in the form of an $\mathrm{AB}$ quartet, as observed for compounds 10a-f. Generally, the pyridine ring in compounds 11a-f shows a unique pattern as a doublet of doublets exists at $7.2 \mathrm{ppm}$, a multiplet (doublet of doublets or doublet of triplets) at 7.8 , a doublet (or a doublet of doublets) at 8.4 and a singlet (or a doublet) at $8.6 \mathrm{ppm}$, and correctly confirm the presence of a pyridine-3-yl substructure. Specifically, in the ${ }^{1} \mathrm{H}-\mathrm{NMR}$ 
spectrum of compound 11e, signals appeared at 7.73 and $7.87 \mathrm{ppm}$ as a doublet corresponding to 4-bromophenyl ring protons. Signals at 7.20 (dd), 7.81 (dt), 8.46 (dd) and 8.66 (d) are assigned as pyridine-3-yl protons. Thus, signals at 4.63 and $8.91 \mathrm{ppm}$ are related to aliphatic $\mathrm{SCH}_{2}$ and triazine $\mathrm{C} 7-\mathrm{H}$ protons respectively. In the ${ }^{13} \mathrm{C}-\mathrm{NMR}$ spectrum of 11e, the aliphatic $\mathrm{SCH}_{2}$ carbon atom appeared at $33.23 \mathrm{ppm}$ and the remaining 13 aromatic carbon atoms are located in the range of 123.64-150.37 ppm and certainly confirms the correct structure of this compound.

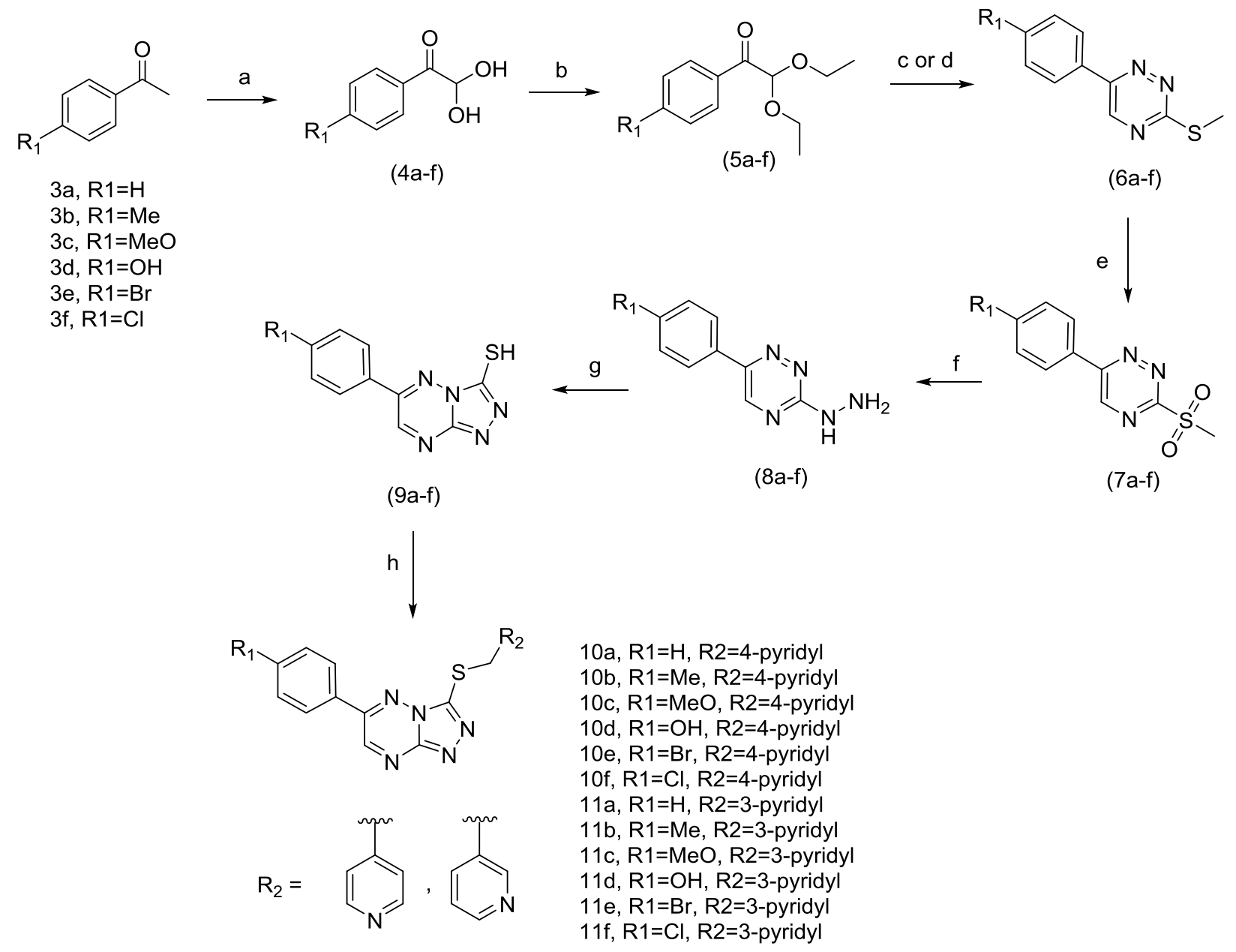

Figure 3. Synthetic scheme of triazolotriazines $10 a$-f and 11a-f. a) $1 . \mathrm{SeO}_{2}, 1$,4-dioxane, reflux 2. $\mathrm{H}_{2} \mathrm{O}$, reflux b) Triethyl orthoformate, PTSA, $\mathrm{CH}_{2} \mathrm{Cl}_{2}$, reflux c) compounds $6 a-c$ and $6 e-f: 1$. Thiosemicarbazide, EtOH, PTSA, rt 2. $\mathrm{CH}_{3}$ l, stirr, rt 3. $\mathrm{AcOH}, 60^{\circ} \mathrm{C}$ d) compound 6 d: methyl hydrazinecarbimidothioate hydroiodide, PTSA, EtOH, rt 2. AcOH, $60^{\circ} \mathrm{C}$, stirr e) Oxone, THF, $\mathrm{H}_{2} \mathrm{O}$, rt f) $\mathrm{NH}_{2} \mathrm{NH}_{2} \cdot \mathrm{H}_{2} \mathrm{O}$, THF, rt g) $\mathrm{CS}_{2}, \mathrm{KOH}$, EtOH, reflux h) $\mathrm{R}_{2} \mathrm{CH}_{2} \mathrm{Cl}, \mathrm{K}_{2} \mathrm{CO}_{3}$, DMSO, rt.

\section{In vitro stability study}


${ }^{13} \mathrm{C}-\mathrm{NMR}$ spectroscopy was used to evaluate the stability of compound $\mathbf{1 1 d}$ in a mixture of deuterated DMSO and chloroform. The solution of compound 11d was stable at room temperature after 4 weeks and any signs of degradation or variations in chemical shifts were not observed (data in Supplementary material).

\section{X-ray crystallography}

X-ray crystallography was performed for a crystal of compound 11c and the results are shown in Figure 4 and presented in Table 1. There is one molecule of 11c in the asymmetric unit, giving rise to four molecules in the unit cell (Figure S1 in supplementary material). The molecules pack together in a zig zag fashion along the c axis (Figure S2 in supplementary material). A series of $\pi-\pi$ interactions between the aromatic rings in adjacent molecules serve to hold the packed structure together. For example, the distance of the centroid of ring $\mathrm{C}(10), \mathrm{C}(11), \mathrm{C}(12), \mathrm{C}(13)$, $\mathrm{C}(14), \mathrm{C}(15)$ to the plane in the adjacent molecule containing the ring $\mathrm{N}(2), \mathrm{N}(3), \mathrm{N}(6), \mathrm{C}(4)$, $\mathrm{C}(5), \mathrm{C}(7)$ is $3.426(2) \AA$, and the distance of the centroid of the ring $\mathrm{N}(2), \mathrm{N}(3), \mathrm{N}(6), \mathrm{C}(4), \mathrm{C}(5)$, $\mathrm{C}(7)$ to this plane is 3.276(2) A. Also the distance of the centroid of the ring $\mathrm{N}(20), \mathrm{C}(18), \mathrm{C}(19)$, $\mathrm{C}(21), \mathrm{C}(22), \mathrm{C}(23)$ to the plane in the adjacent molecule containing the ring $\mathrm{N}(20), \mathrm{C}(18)$, $\mathrm{C}(19), \mathrm{C}(21), \mathrm{C}(22), \mathrm{C}(23)$ is 3.432(2) $\AA$. Notably, the distance of the centroid of the ring $\mathrm{N}(2)$, $\mathrm{N}(3), \mathrm{N}(6), \mathrm{C}(4), \mathrm{C}(5), \mathrm{C}(7)$ to the plane in the adjacent molecule containing the ring $\mathrm{N}(2), \mathrm{N}(8)$, $\mathrm{N}(9), \mathrm{C}(1), \mathrm{C}(7)$ is $3.245(2) \AA$. (Figure S3 in supplementary material) Data have been deposited at the Cambridge Crystallographic Data Centre, deposit code: CCDC 1853261. 


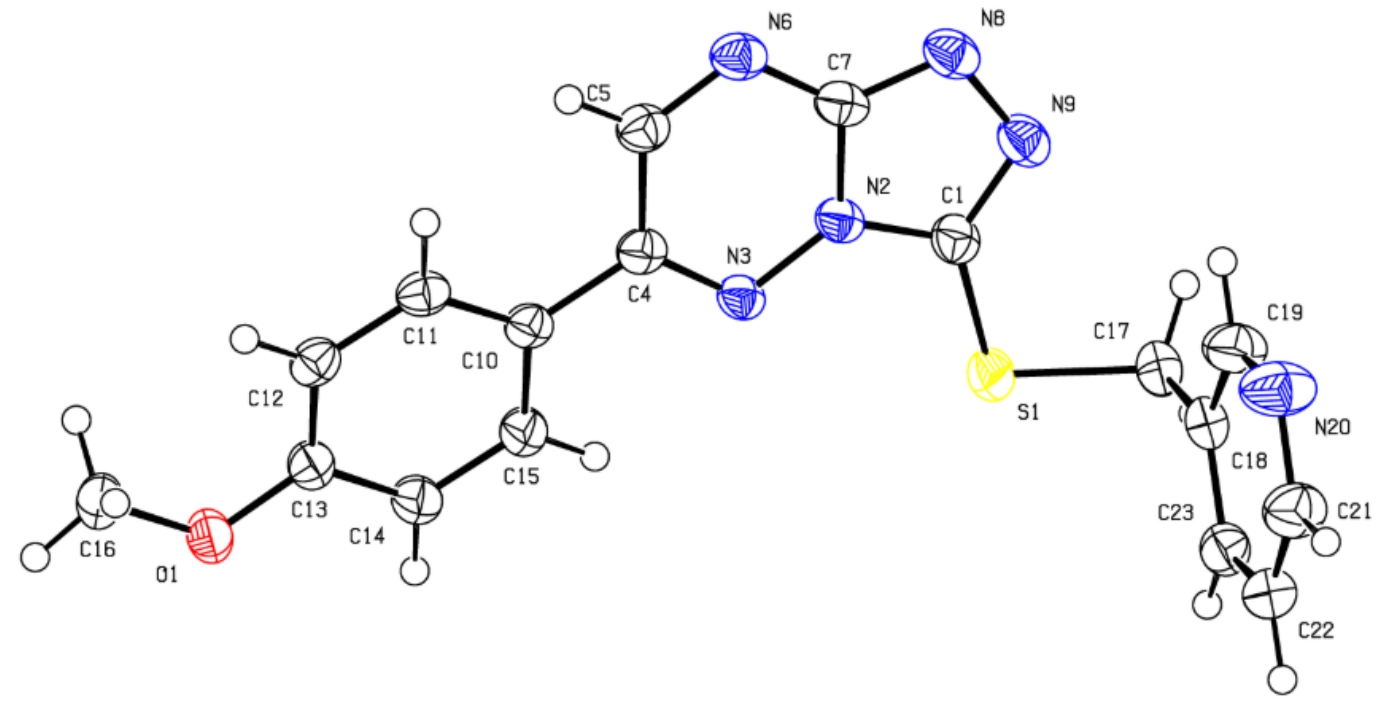

Figure 4. ORTEP structure of compound 11c; Asymmetric unit with labelling scheme.

Table 1. Crystallographic data for 11c at $150 \mathrm{~K}$.

\begin{tabular}{cc}
\hline Formula & $\mathrm{C}_{17} \mathrm{H}_{14} \mathrm{~N}_{6} \mathrm{O}_{1} \mathrm{~S}_{1}$ \\
$M_{\mathrm{r}}$ & 350.40 \\
Crystal system & monoclinic \\
Space group & $P 2{ }_{1} / n$ \\
$\mathrm{Z}$ & 4 \\
$a / \AA$ & $4.77748(8)$ \\
$b / \AA$ & $18.6830(5)$ \\
$c / \AA$ & $17.9296(5)$ \\
$\beta /{ }^{\circ}$ & $94.1914(18)$ \\
$V / \AA^{3}$ & $1596.07(7)$ \\
$\rho{ }^{3}$ calc/g cm & \\
Crystal habit & 1.458 \\
Crystal dimensions $/ \mathrm{mm}$ & yellow plate \\
Radiation & $0.03 \times 0.06 \times 0.21$ \\
T/K & $\mathrm{Cu} \mathrm{K \alpha}$ \\
$\mu / \mathrm{mm}^{-1}$ & 150 \\
no. parameters & 1.967 \\
$\mathrm{R}(F), \mathrm{Rw}^{-3}(F)$ & 2684,226 \\
\hline
\end{tabular}




\section{HGF-induced proliferation assay}

The inhibitory effect of compounds on HGF-induced cell proliferation was evaluated against four cancer cell lines, including A549, HepG2 and MCF7 as high c-Met expressing cell lines, and against MDA-MB231 as a low c-Met expressing cell line and the results are summarized in Table 2. Crizotinib, as a selective c-Met kinase inhibitor, was used as a reference drug in this study and the cancer cells were treated with recombinant human Hepatocyte Growth Factor (HGF) to induce c-Met kinase expression and activation. As the data in Table 2 indicate, most compounds exhibited good to excellent cytotoxicity with $\mathrm{IC}_{50}$ values ranging from 0.71 to $24.29 \mu \mathrm{M}$ against four cancer cells. In general, all target compounds were more potent against A549 and HepG2 compared to the other two cell lines, MCF7 and MDA-MB231. $\mathrm{IC}_{50}$ values of the target compounds on $\mathrm{A} 549$ range from 0.74 to $5.25 \mu \mathrm{M}$, but none of them showed more potency than the reference drug crizotinib $(0.32 \mu \mathrm{M})$. The most potent compound on A549 cell line was determined to be compound $\mathbf{1 1 b}$, with an $\mathrm{IC}_{50}$ value of $0.74 \mu \mathrm{M}$. Interestingly, the data on $\mathrm{HepG} 2$ are the most promising since $\mathrm{IC}_{50}$ values range from 0.71 to $3.25 \mu \mathrm{M}$ and two of the compounds displayed more cytotoxicity than crizotinib. Notably, compounds $\mathbf{1 0 b}$ and $11 \mathrm{e}$ with $\mathrm{IC}_{50}$ values of 0.74 and $0.71 \mu \mathrm{M}$ respectively on HepG2 cells, showed 2.1 and 2.2-fold more potency than crizotinib $\left(\mathrm{IC}_{50}=1.57 \mu \mathrm{M}\right)$. Moreover, the target compounds showed acceptable cytotoxicity on MCF7 cancer cell lines and their $\mathrm{IC}_{50}$ values were in the range 9.46-21.17 $\mu \mathrm{M}$. None of the target molecules were more cytotoxic than crizotinib $\left(\mathrm{IC}_{50}=3.028 \mu \mathrm{M}\right)$ on $\mathrm{MCF} 7$ cells but again compound $10 \mathrm{~b}$ was shown to have more cytotoxicity than the others with an $\mathrm{IC}_{50}$ value of $9.46 \mu \mathrm{M}$. The target compounds exhibited mild to moderate potency on MDA-MB-231 as a low expressing c-Met cell line. In all, HepG2 was the most sensitive cancer cell line against target molecules 10a-e and 11a-e. Substituent variations on the 6-aryl ring by methyl, methoxy, hydroxyl, bromine and chlorine groups displayed minimal impact on the cell proliferation results, so that no correlation could be found to relate structure and activity of these compounds. This finding can also be confirmed by docking calculations, in which the 6-aryl ring is oriented towards the solvent accessible region and there is not any contact between groups attached to the 
6-aryl ring and the c-Met active site. There is no improvement or significant difference in the biological effects of the 4-pyridyl- and 3-pyridyl linked triazolotriazine compounds. On the other hand, the type of substitution of the pyridine ring has not changed the $\mathrm{IC}_{50}$ values on all cell lines significantly. This fact is clarified and discussed in the docking part of the discussion.

Table 2. Structure and $\mathrm{IC}_{50}$ values of compounds 10a-f and 11a-f in HGF-induced cell proliferation assay.

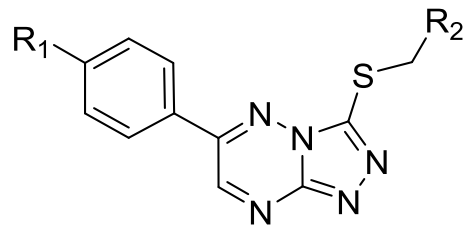

\begin{tabular}{|c|c|c|c|c|c|c|}
\hline \multirow[t]{2}{*}{ Compd. } & \multirow[t]{2}{*}{$\mathrm{R}_{1}$} & \multirow[t]{2}{*}{$\mathrm{R}_{2}$} & \multicolumn{3}{|c|}{$\mathrm{IC}_{50}(\mu \mathrm{M})$} & \multirow[b]{2}{*}{ MDA-MB-231 } \\
\hline & & & A549 & HepG2 & MCF7 & \\
\hline 10a & $\mathrm{H}$ & 4-pyridyl & $2.02 \pm 0.03$ & $1.27 \pm 0.02$ & $16.43 \pm 1.09$ & $9.65 \pm 1.15$ \\
\hline $10 \mathrm{~b}$ & $\mathrm{Me}$ & 4-pyridyl & $1.14 \pm 0.09$ & $0.74 \pm 0.07 *$ & $9.46 \pm 1.10$ & $24.29 \pm 1.20$ \\
\hline $10 \mathrm{c}$ & $\mathrm{MeO}$ & 4-pyridyl & $5.20 \pm 0.05$ & $1.10 \pm 0.03$ & $9.64 \pm 1.06$ & $6.09 \pm 1.05$ \\
\hline 10d & $\mathrm{OH}$ & 4-pyridyl & $4.68 \pm 0.04$ & $1.27 \pm 0.02$ & $10.07 \pm 1.06$ & $11.15 \pm 1.06$ \\
\hline $10 \mathrm{e}$ & $\mathrm{Br}$ & 4-pyridyl & $5.05 \pm 0.04$ & $1.27 \pm 0.03$ & $10.75 \pm 1.07$ & $6.36 \pm 1.13$ \\
\hline $10 f$ & $\mathrm{Cl}$ & 4-pyridyl & $5.25 \pm 0.03$ & $1.47 \pm 0.02$ & $21.17 \pm 1.21$ & $16.18 \pm 1.15$ \\
\hline $11 a$ & $\mathrm{H}$ & 3-pyridyl & $3.11 \pm 0.04$ & $1.11 \pm 0.02$ & $19.47 \pm 1.10$ & $11.16 \pm 1.12$ \\
\hline 11b & $\mathrm{Me}$ & 3-pyridyl & $0.74 \pm 0.05$ & $1.02 \pm 0.03$ & $9.76 \pm 1.06$ & $11.55 \pm 1.06$ \\
\hline 11c & $\mathrm{MeO}$ & 3-pyridyl & $1.76 \pm 0.04$ & $3.25 \pm 0.04$ & $10.19 \pm 1.03$ & $10.07 \pm 1.07$ \\
\hline 11d & $\mathrm{OH}$ & 3-pyridyl & $1.43 \pm 0.04$ & $2.11 \pm 0.07$ & $10.19 \pm 1.05$ & $11.91 \pm 1.06$ \\
\hline $11 \mathrm{e}$ & $\mathrm{Br}$ & 3-pyridyl & $2.33 \pm 0.06$ & $0.71 \pm 0.04 *$ & $12.55 \pm 1.09$ & $11.59 \pm 1.07$ \\
\hline $11 f$ & $\mathrm{Cl}$ & 3-pyridyl & $2.78 \pm 0.06$ & $1.01 \pm 0.02$ & $17.89 \pm 1.08$ & $9.48 \pm 1.09$ \\
\hline Crizotinib & & & $0.32 \pm 0.06$ & $1.57 \pm 0.03$ & $3.028 \pm 1.06$ & $1.99 \pm 1.08$ \\
\hline
\end{tabular}

The data are presented as mean \pm SEM and each experiment was performed in triplicate.

Bold values show the $\mathrm{IC}_{50}$ values of the target compounds lower than the values of crizotinib (also marked by an asterisk) or the most promising compounds in each column.

\section{Caspase 3/7 Activity Assay}

Caspases pertain to cysteine-aspartic proteases, promote controlled cell death or apoptosis. They modulate both intrinsic and extrinsic apoptotic pathways. Activation of caspases are mediated by proteolytic cleavage and finally leading to cell death during the apoptotic process. Impairment of apoptosis contribute in cancer development and progression. According to studies, the amount of caspases in cancer cells is reduced compared to normal cells. 
The compounds were tested at $20 \mu \mathrm{M}$ for 24 hours for viability, cytotoxicity and caspase $3 / 7$ activity which is a marker of apoptosis in MCF7, MDA-MB-231, HepG2 and A549 cells and the data are illustrated in Figure 5. Accordingly, the compounds showed high cytotoxicity while caspase 3/7 activity was decreased. These results suggest that compounds are fast acting necrotic agents within $24 \mathrm{~h}$ and do not induce apoptosis through caspase 3/7 activity. These data also confirmed the results obtained by MTT assay (Table 2) in which target compounds had the most cytotoxic effect on HepG2 cells.

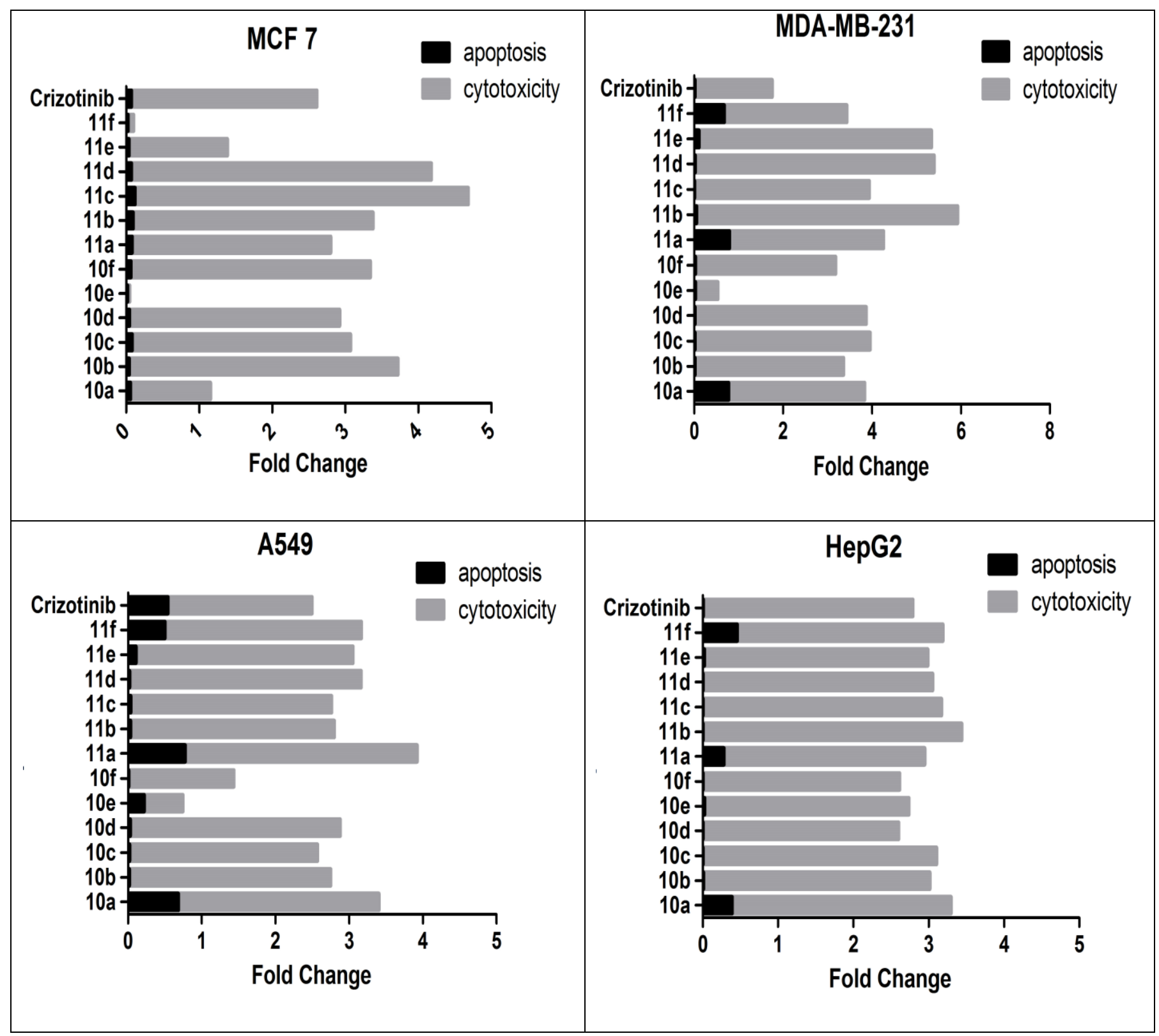

Figure 5. Extent of cytotoxicity and apoptosis mediated by Caspase 3/7 activity on MCF7, A549, HepG2 and MDA-MB-231 cell lines. Target compounds were tested at $20 \mu \mathrm{M}$ concentration. 


\section{c-Met kinase inhibition assay}

All compounds displayed significant c-Met kinase inhibition potential which were more than the values of crizotinib at $5,0.5,0.05 \mu \mathrm{M}$ and $0.5 \mathrm{nM}$ concentrations and the data are summarized in Table 3 , as percent inhibition of c-Met kinase activity. All compounds showed complete inhibition of c-Met kinase activity at $20 \mu \mathrm{M}$, while crizotinib has $91 \%$ inhibition of c-Met kinase activity at this concentration (data not shown). At $5 \mu \mathrm{M}$ concentration, all compounds had higher than $90 \%$ inhibition of c-Met, while compounds 10a and 11d showed the highest percentage inhibitions of $96.8 \%$ and $97.7 \%$, respectively, which is higher than crizotinib (92.0\%). At $0.5 \mu \mathrm{M}$ concentration of target compounds, the percentage inhibition of c-Met was evaluated to be more than $80 \%$ for all compounds and interestingly, compounds 10a $(85.9 \%), \mathbf{1 0 b}(84.2 \%), \mathbf{1 1 b}(84.4 \%), \mathbf{1 1 e}(87.7 \%)$ and $\mathbf{1 1 f}(86.1 \%)$ were shown to be more potent than crizotinib (83.0\%) at this concentration. Surprisingly, the evaluation of c-Met kinase activity at lower concentration $(0.05 \mu \mathrm{M})$ of target compounds, showed that compounds 10a $(79.3 \%), \mathbf{1 0 b}(80.8 \%), \mathbf{1 0 c}$ (77.6\%), 10e (77.9\%), 11b (80.9\%), 11e (79.1\%) and $\mathbf{1 1 f}(81.4 \%)$ have more inhibition potential of c-Met kinase activity than crizotinib (76.0\%). Finally at $0.5 \mathrm{nM}$ concentration, all the compounds exhibited a higher percent inhibition of c-Met kinase activity than crizotinib. Interestingly, all compounds displayed $\mathrm{IC}_{50}$ values in the range of 3.9-11.1 $\mathrm{nM}$ for c-Met kinase inhibition, clearly confirming that all compounds are more potent than crizotinib $\left(\mathrm{IC}_{50}=11.1 \mathrm{nM}\right)$.

As the data in Table 3 indicate, inclusion of the substituted pyridine ring (3- or 4-pyridyl) has not induced a significant or regular difference in potency between compounds 10a-f and their equivalent derivatives 11a-f. Correspondingly, the potency difference between the compounds is largely dependent on the type of substituted group at the para position of the phenyl ring. On the other hand, methyl- and brominesubstituted compounds $\mathbf{1 0 b}, \mathbf{1 0 e}, \mathbf{1 1 b}$ and $\mathbf{1 1 e}$ are the most potent ones in this series.

The obtained results confirmed our original hypothesis about high potency of thiomethylpyridine linked triazolotriazines to inhibit c-Met kinase activity and successfully suggest that all compounds and especially $\mathbf{1 0 b}$ are recommended drug candidates for further in vivo and in vitro evaluations in the future. 
Table 3. Percent inhibition of c-Met kinase activity by target compounds 10a-f and 11a-f at four different concentrations.

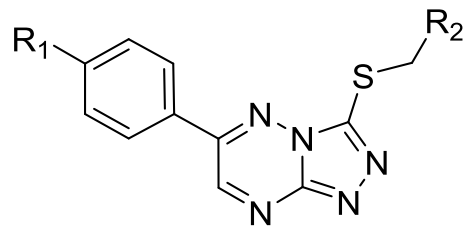

\begin{tabular}{cccccccc}
\hline Compd. & $\mathrm{R} 1$ & $\mathrm{R} 2$ & \multicolumn{5}{c}{$\% \mathrm{c}-\mathrm{M}$ et inhibition $\pm \mathrm{SEM}$} \\
\cline { 3 - 6 } & & & $5 \mu \mathrm{M}$ & $0.5 \mu \mathrm{M}$ & $0.05 \mu \mathrm{M}$ & $0.5 \mathrm{nM}$ & $\mathrm{IC}_{50}(\mathrm{nM})$ \\
\hline $\mathbf{1 0 a}$ & $\mathrm{H}$ & 4-pyridyl & $96.8 \pm 0.9$ & $\mathbf{8 5 . 9} \pm \mathbf{3 . 3 5}$ & $\mathbf{7 9 . 3} \pm \mathbf{1 . 7}$ & $\mathbf{1 9 . 0} \pm \mathbf{1 . 7}$ & $6.2 \pm 0.07$ \\
$\mathbf{1 0 b}$ & $\mathrm{Me}$ & 4-pyridyl & $93.9 \pm 1.3$ & $\mathbf{8 4 . 2} \pm \mathbf{4 . 6 2}$ & $\mathbf{8 0 . 8} \pm \mathbf{1 . 8}$ & $\mathbf{2 3 . 8} \pm \mathbf{3 . 6}$ & $4.6 \pm 0.10$ \\
$\mathbf{1 0 c}$ & $\mathrm{MeO}$ & 4-pyridyl & $94.6 \pm 3.2$ & $81.1 \pm 5.32$ & $\mathbf{7 7 . 6} \pm \mathbf{1 . 9}$ & $\mathbf{1 7 . 1} \pm \mathbf{3 . 1}$ & $8.0 \pm 0.12$ \\
$\mathbf{1 0 d}$ & $\mathrm{OH}$ & 4-pyridyl & $95.8 \pm 1.7$ & $83.3 \pm 2.44$ & $74.4 \pm 1.2$ & $\mathbf{2 3 . 0} \pm \mathbf{2 . 5}$ & $6.5 \pm 0.07$ \\
$\mathbf{1 0 e}$ & $\mathrm{Br}$ & 4-pyridyl & $92.1 \pm 2.3$ & $81.1 \pm 3.37$ & $\mathbf{7 7 . 9} \pm \mathbf{3 . 0}$ & $\mathbf{2 9 . 6} \pm \mathbf{1 . 7}$ & $3.9 \pm 0.10$ \\
$\mathbf{1 0 f}$ & $\mathrm{Cl}$ & 4-pyridyl & $93.1 \pm 1.9$ & $83.5 \pm 1-17$ & $74.8 \pm 3.4$ & $\mathbf{1 2 . 1} \pm \mathbf{2 . 9}$ & $11.1 \pm 0.08$ \\
$\mathbf{1 1 a}$ & $\mathrm{H}$ & 3-pyridyl & $96.8 \pm 1.4$ & $83.2 \pm 2.42$ & $73.9 \pm 2.4$ & $\mathbf{1 6 . 6} \pm \mathbf{2 . 3}$ & $9.3 \pm 0.07$ \\
$\mathbf{1 1 b}$ & $\mathrm{Me}$ & 3-pyridyl & $93.5 \pm 1.0$ & $\mathbf{8 4 . 4} \pm \mathbf{4 . 5 8}$ & $\mathbf{8 0 . 9} \pm \mathbf{1 . 6}$ & $\mathbf{2 0 . 3} \pm \mathbf{3 . 5}$ & $5.6 \pm 0.10$ \\
$\mathbf{1 1 c}$ & $\mathrm{MeO}$ & 3-pyridyl & $94.4 \pm 1.0$ & $80.0 \pm 2.84$ & $71.7 \pm 3.5$ & $\mathbf{2 3 . 0} \pm \mathbf{2 . 9}$ & $7.8 \pm 0.09$ \\
$\mathbf{1 1 d}$ & $\mathrm{OH}$ & 3-pyridyl & $97.7 \pm 0.8$ & $79.5 \pm 2.32$ & $75.4 \pm 2.8$ & $\mathbf{1 4 . 6} \pm \mathbf{1 . 7}$ & $10.1 \pm 0.09$ \\
$\mathbf{1 1 e}$ & $\mathrm{Br}$ & 3-pyridyl & $90.2 \pm 1.3$ & $\mathbf{8 7 . 7} \pm \mathbf{2 . 0 2}$ & $\mathbf{7 9 . 1} \pm \mathbf{3 . 3}$ & $\mathbf{1 8 . 2} \pm \mathbf{1 . 0}$ & $6.4 \pm 0.08$ \\
$\mathbf{1 1 f}$ & $\mathrm{Cl}$ & 3-pyridyl & $92.4 \pm 1.4$ & $\mathbf{8 6 . 1} \pm \mathbf{2 . 5 4}$ & $\mathbf{8 1 . 4} \pm \mathbf{2 . 9}$ & $\mathbf{1 5 . 4 \pm 1 . 0}$ & $6.7 \pm 0.08$ \\
Crizotinib & & & $92.0 \pm 2.2$ & $83.0 \pm 2.61$ & $76.0 \pm 1.7$ & $11.4 \pm 1.4$ & $11.1 \pm 0.09$ \\
\hline
\end{tabular}

Bold values are \%inhibition values higher than the value of crizotinib.

\section{Molecular docking study}

To explore the binding mode of the target compounds and to reach conclusions about the structure-activity relationship, molecular docking was performed for the two compounds, 10c and 11c as 4- and 3-pyridyl-linked triazolotriazines, respectively. Accordingly, PDB code 3ZXZ was used for an in silico study and our docking protocol was validated by the docking of cocrystal ligand PF-04217903 into the ATP-binding site of c-Met tyrosine kinase. The results of docking for the two compounds, 10c and 11c, are presented in Figure 6, and Figures S4 and S5 
in the Supplementary material. As illustrated in Figure 6, both compounds have a very similar binding conformation so that the two compounds are almost superimposed on each other. Essentially, the triazolotriazine ring forms a hydrogen bond with Asp1222 and a $\pi-\pi$ stacking interaction with Tyr1230, which are important in the exquisite c-Met kinase selectivity and potency. The pyridyl moiety occupies the hinge region and its nitrogen atom forms a crucial hydrogen bond with Met1160. The presence of a hydrogen-bond acceptor atom at this region of inhibitor has been implied in many SAR studies of c-Met kinase inhibitors [22]. As shown in Figures S4 and S5, this hydrogen bond has distances of 1.94 and $1.89 \AA$, respectively for compounds 10c and 11c. Furthermore, $\pi-\pi$ stacking interaction occurs between Tyr1230 and $p$ methoxyphenyl ring. There are also several pi-alkyl interactions between bound inhibitors and aliphatic amino acids in the active site such as the alanine, valine and leucine residues. 
a)

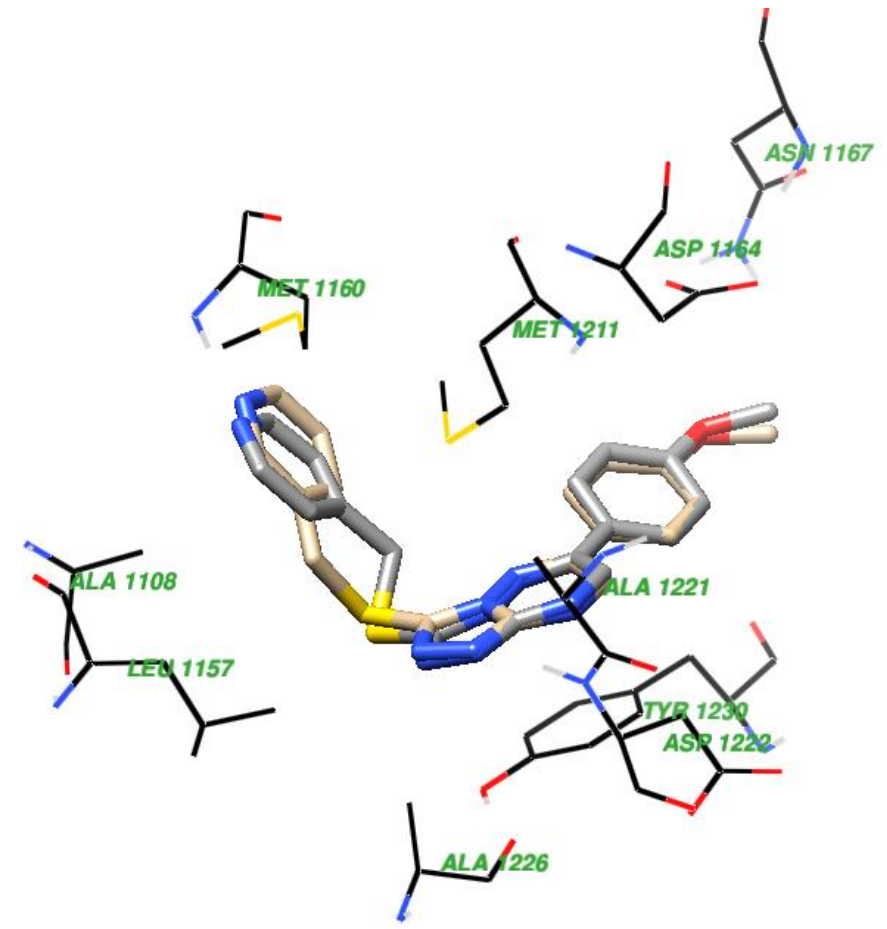

b)

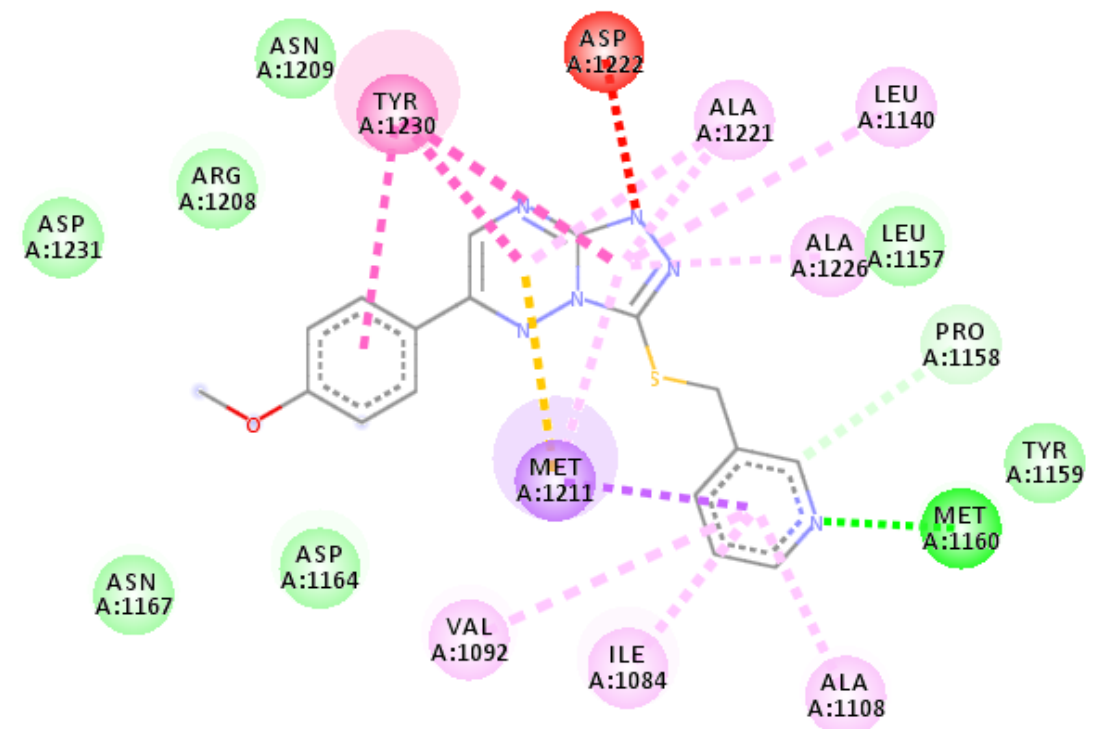

Interactions

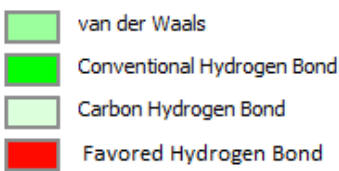

Pi-Sigma

Pi-Sulfur

Pi-Pi Stacked

Pi-Alkyl 
Figure 6. a) Binding conformation of compounds 10c (gray) and 11c (brown) in the ATP-binding site of c-Met kinase. b) 2D map of bound conformation of 11c in the ATP-binding site of c-Met kinase.

\section{Conclusion}

In summary, a series of compounds for use as c-Met kinase inhibitors were designed, synthesized and characterized by NMR, Mass spectrometry and X-ray crystallography. Their cytotoxicity was evaluated on A549, HepG2, MCF7 and MDA-MB-231 cancer cell lines. Most of the target compounds exhibited good to excellent cytotoxicity on A549 and HepG2 cell lines with $\mathrm{IC}_{50}$ values ranging from 0.71 to $5.25 \mu \mathrm{M}$. Interestingly, compounds $\mathbf{1 0 b}$ and 11e displayed more cytotoxicity than the reference drug crizotinib on HepG2 cells with IC50 of 0.74 and $0.71 \mu \mathrm{M}$ respectively. Evaluation of c-Met kinase activity of target compounds showed that all compounds are more potent than crizotinib with IC50 values in the range of 3.9-11.1 nM. Finally, compound 10b was introduced as a lead compound with $\mathrm{IC}_{50}$ value of $4.6 \mathrm{nM}$ for c-Met kinase activity.

\section{Future Perspective}

Due to the emerge of new cancer types and resistance phenomenon to antitumor drugs, key biologic macromolecules involved in cancer progression have attracted much attention in drug discovery. c-Met tyrosine kinase receptor is one of the crucial targets responsible for tumor progression and migration and several studies have disclosed the triazolotriazines as a privileged scaffold for c-Met kinase inhibition. In the current study, novel triazolotriazine analogs were introduced as new leads for future drug development process as potent c-Met kinase inhibitors.

\section{Summary Points}

Design \& synthesis of target compounds

- A series of compounds were designed, synthesized and biologically evaluated as c-Met tyrosine kinase inhibitors.

- Target compounds were characterized by ${ }^{1} \mathrm{H}$ and ${ }^{13} \mathrm{C}-\mathrm{NMR}$, Mass spectrometry and X-ray crystallography.

Biological studies 
- Compounds $\mathbf{1 0 b}$ and 11e were more cytotoxic than crizotinib on $\mathrm{HepG} 2$ cells with $\mathrm{IC}_{50}$ values of 0.74 and $0.71 \mu \mathrm{M}$ respectively.

- All of the compounds were more potent than crizotinib in c-Met kinase inhibition assay.

- Compound 10b was introduced as a lead compound with $\mathrm{IC}_{50}$ of $4.6 \mathrm{nM}$ for c-Met kinase.

\section{References}

Papers of special note have been highlighted as: * of interest; ** of considerable interest

1. Trusolino L, Bertotti A, Comoglio PM. MET signalling: principles and functions in development, organ regeneration and cancer. Nat Rev Mol Cell Bio 11(12), 834-848 (2010).

** this article fully describes role of c-Met signaling in cancer progression.

2. Birchmeier C, Birchmeier W, Gherardi E, Vande Woude GF. Met, metastasis, motility and more. Nat Rev Mol Cell Bio 4(12), 915-925 (2003).

3. Cui JJ. Targeting Receptor Tyrosine Kinase MET in Cancer: Small Molecule Inhibitors and Clinical Progress. J Med Chem 57(11), 4427-4453 (2014).

* this article describes a comprehensive SAR on c-Met kinase inhibitors.

4. Ma YC, Sun GQ, Chen DQ et al. Design and Optimization of a Series of 1-Sulfonylpyrazolo[4,3b]pyridines as Selective c-Met Inhibitors. J Med Chem 58(5), 2513-2529 (2015).

5. Albrecht BK, Harmange JC, Bauer D et al. Discovery and optimization of triazolopyridazines as potent and selective inhibitors of the c-Met kinase. J Med Chem 51(10), 2879-2882 (2008).

** this article reviews triazolotriazine core scaffold for c-Met kinase inhibition

6. Buchanan SG, Hendle J, Lee PS et al. SGX523 is an exquisitely selective, ATP-competitive inhibitor of the MET receptor tyrosine kinase with antitumor activity in vivo. Mol Cancer Ther 8(12), 3181-3190 (2009).

7. Chen F, Wang Y, Ai J et al. O-Linked Triazolotriazines: Potent and Selective c-Met Inhibitors. Chemmedchem 7(7), 1276-1285 (2012).

* this article is helpful for its synthetic part.

8. Cui JJ, Mctigue M, Nambu M et al. Discovery of a Novel Class of Exquisitely Selective Mesenchymal-Epithelial Transition Factor (c-MET) Protein Kinase Inhibitors and Identification of the Clinical Candidate 2-(4-(1-(Quinolin-6-ylmethyl)-1H-[1,2,3]triazolo[4,5-b]pyrazin-6-yl)-1Hpyrazol-1-yl)ethanol (PF-04217903) for the Treatment of Cancer. J Med Chem 55(18), 8091-8109 (2012).

9. Rigaku. Xcalibur/SuperNova CCD System (formerly Oxford Diffraction), CrysAlisPro Software System, Version 1.171.36.21, Rigaku Ltd., UK, . (2012).

10. Palatinus L, Chapuis G. SUPERFLIP - a computer program for the solution of crystal structures by charge flipping in arbitrary dimensions. J Appl Crystallogr 40 786-790 (2007).

11. Betteridge PW, Carruthers JR, Cooper RI, Prout K, Watkin DJ. CRYSTALS version 12: software for guided crystal structure analysis. J Appl Crystallogr 36 1487-1487 (2003).

12. Macrae CF, Bruno IJ, Chisholm JA et al. Mercury CSD 2.0 - new features for the visualization and investigation of crystal structures. J App/ Crystallogr 41 466-470 (2008).

13. Jia H, Dai GX, Weng JY et al. Discovery of (S)-1-(1-(Imidazo[1,2-a]pyridin-6-yl)ethyl)-6-(1-methyl$1 \mathrm{H}$-pyrazol-4-yl)-1H-[1,2,3]triazolo[4,5-b]pyrazine (Volitinib) as a Highly Potent and Selective Mesenchymal-Epithelial Transition Factor (c-Met) Inhibitor in Clinical Development for Treatment of Cancer. J Med Chem 57(18), 7577-7589 (2014). 
14. Liao WK, Xu C, Ji XH et al. Design and optimization of novel 4-(2-fluorophenoxy)quinoline derivatives bearing a hydrazone moiety as c-Met kinase inhibitors. Eur J Med Chem 87 508-518 (2014).

15. Fujita $\mathrm{H}$, Miyadera $\mathrm{K}$, Kato $\mathrm{M}$ et al. The Novel VEGF Receptor/MET-Targeted Kinase Inhibitor TAS-115 Has Marked In Vivo Antitumor Properties and a Favorable Tolerability Profile. Mol Cancer Ther 12(12), 2685-2696 (2013).

16. Antoine M, Gerlach M, Gunther E et al. A Convenient Synthesis of Novel 2,8-Disubstituted Pyrido[3,4-b]pyrazines Possessing Biological Activity. Synthesis-Stuttgart 44(1), 69-82 (2012).

17. Zhan ZS, Peng X, Liu QF et al. Discovery of 6-(difluoro(6-(4-fluorophenyl)-[1,2,4]triazolo[4,3-b] $[1,2,4]$ triazin-3-yl)methyl)quinoline as a highly potent and selective c-Met inhibitor. Eur J Med Chem 116 239-251 (2016).

18. K.J. Doyle GPJ, M.G.N. Russell, S. Bruckner, J.A. Macritchie, J. Peach,. Compounds, Compositions and Methods Comprising Heteroaromatic Derivatives. Google Patents (2009).

19. Irannejad H, Kebriaieezadeh A, Zarghi A et al. Synthesis, docking simulation, biological evaluations and 3D-QSAR study of 5-Aryl-6-(4-methylsulfonyl)-3-(metylthio)-1,2,4-triazine as selective cyclooxygenase-2 inhibitors. Bioorgan Med Chem 22(2), 865-873 (2014).

20. El-Sayed WA, Nassar IF, Abdel-Rahman AaH. Synthesis and Antitumor Activity of New 1,2,4Triazine and $[1,2,4]$ Triazolo[4,3-b][1,2,4]triazine Derivatives and Their Thioglycoside and Acyclic C-Nucleoside Analogs. J Heterocyclic Chem 48(1), 135-143 (2011).

21. Dadashpour S, Kucukkilinc TT, Tan OU, Ozadali K, Irannejad H, Emami S. Design, Synthesis and In Vitro Study of 5,6-Diaryl-1,2,4-triazine-3-ylthioacetate Derivatives as COX-2 and beta-Amyloid Aggregation Inhibitors. Arch Pharm 348(3), 179-187 (2015).

22. Tai WT, Lu T, Yuan HL et al. Pharmacophore modeling and virtual screening studies to identify new c-Met inhibitors. J Mol Model 18(7), 3087-3100 (2012).

** this article implies the importance of hydrogen bondong of an $\mathrm{h}$-bond acceptor with Met1160 in the active site. 